\title{
Diversifying Isoprenoid Platforms via Atypical Carbon Substrates and Non-model Microorganisms
}

\author{
David N. Carruthers ${ }^{1,2}$ and Taek Soon Lee ${ }^{1,2 *}$ \\ ${ }^{1}$ Joint BioEnergy Institute, Emeryville, CA, United States, ${ }^{2}$ Biological Systems and Engineering Division, Lawrence Berkeley \\ National Laboratory, Berkeley, CA, United States
}

Isoprenoid compounds are biologically ubiquitous, and their characteristic modularity has afforded products ranging from pharmaceuticals to biofuels. Isoprenoid production has been largely successful in Escherichia coli and Saccharomyces cerevisiae with metabolic engineering of the mevalonate (MVA) and methylerythritol phosphate (MEP) pathways coupled with the expression of heterologous terpene synthases. Yet conventional microbial chassis pose several major obstacles to successful commercialization including the affordability of sugar substrates at scale, precursor flux limitations, and intermediate feedback-inhibition. Now, recent studies have challenged typical isoprenoid paradigms by expanding the boundaries of terpene biosynthesis and using non-model organisms

Edited by: João M. P. Jorge, Universidade Nova de Lisboa,

Portugal

Reviewed by:

Chonglong Wang,

Soochow University, China

Guang Zhao,

Shandong University, China

${ }^{*}$ Correspondence:

Taek Soon Lee

tslee@lbl.gov

Specialty section:

This article was submitted to Microbiotechnology,

a section of the journal

Frontiers in Microbiology

Received: 07 October 2021 Accepted: 10 November 2021 Published: 02 December 2021

Citation:

Carruthers DN and Lee TS (2021) Diversifying Isoprenoid Platforms via Atypical Carbon Substrates and Non-model Microorganisms. Front. Microbiol. 12:791089. doi: 10.3389/fmicb.2021.791089 including those capable of metabolizing atypical $\mathrm{C} 1$ substrates. Conversely, investigations of non-model organisms have historically informed optimization in conventional microbes by tuning heterologous gene expression. Here, we review advances in isoprenoid biosynthesis with specific focus on the synergy between model and non-model organisms that may elevate the commercial viability of isoprenoid platforms by addressing the dichotomy between high titer production and inexpensive substrates.

Keywords: isoprenoids, metabolic engineering, synthetic biology, non-model organisms, C1 metabolism, terpenes

\section{INTRODUCTION}

Isoprenoids are ubiquitous across all domains of life and span a wide and varied range of natural products. Isoprenoids are characterized by condensation of the five carbon precursor molecules isopentenyl diphosphate (IPP) and dimethylallyl diphosphate (DMAPP), which are typically generated through either the mevalonate (MVA) or methylerythritol phosphate (MEP) pathways. The ease with which specialized synthases and cytochromes can conjugate or decorate these precursors has led to a uniquely diverse class of chemicals. Estimates of natural isoprenoid compounds in the last several decades have steadily increased from 20,000 (Chappell, 1995) to over 70,000 (Moser and Pichler, 2019). The advent of advanced sequencing, -omics, and bioinformatics technologies coupled with protein structural software and flux balance analyses have facilitated a veritable revolution in synthetic biology and assured the continued elucidation of isoprenoid compounds through bioprospecting and biosynthetic efforts.

Isoprenoids serve a number of critical roles both as primary and secondary metabolites. Primary metabolites are essential to cell survival and propagation. They include carotenoids 
that serve as auxiliary molecules for photoprotection and antioxidants (carotene, lycopene, lutein, and zeaxanthin) as well as sterols that help maintain membrane structure. Other isoprenoids function as components of dolichols, quinones, and essential proteins that aid in glycosylation and electron transport (Chappell, 1995). Secondary isoprenoid metabolites impart a non-essential benefit to cells usually by providing some defensive benefit or, in higher plants, hormone signaling. As for isoprenoids, these include pigments, fragrances, essential oils, and defensive chemicals that are most prominent in higher plants. Many secondary metabolites have attracted particular interest due to their applications as pharmaceuticals [e.g., artemisinin (Ro et al., 2006) and pacilitaxel (Biggs et al., 2016)], nutraceuticals, biofuels [e.g., isoprenol (Kang et al., 2019), prenol (Zheng et al., 2013), bisabolene, and limonene (AlonsoGutierrez et al., 2013)], and cosmetics (Schempp et al., 2018). Hybrid technologies have capitalized on isoprenoid versatility through semi-synthetic approaches to generate elastomers (Della Monica and Kleij, 2020). Collectively, the bioproduction of these chemicals has enabled access to multibillion dollar chemical markets.

Microbial pathway engineering has proven especially successful in Escherichia coli and Saccharomyces cerevisiae, which have produced many of the aforementioned isoprenoid compounds. E. coli and S. cerevisiae maintain certain metabolic advantages including a fast growth phenotype, historical breadth of knowledge, ease of transformation and hence heterologous protein expression, substrate specificity, and published successes of bioproduction (Vickers et al., 2017; Ward et al., 2018). These advantages are complemented by specialized synthetic biology strategies that enable tuning of ribosome binding site and promoter strength, codon optimization of heterologous proteins, protein fusions, and the knocking out of competing pathways. In recent years, this has been accomplished by systematic gene downregulation using regulatable CRISPR interference systems (Kim et al., 2016; Tian et al., 2019) that express a modified dCas9 protein for fine-tuning of the overall pathway and optimization of target production. Furthermore, E. coli endogenously generates isoprenoids through the MEP pathway while $S$. cerevisiae utilizes its native MVA pathway, together enabling researchers to combine synthetic biological toolkits with the abundance of information of these strains to facilitate high-titer isoprenoid production. As for downstream isoprenoid functionalization, these metabolic chassis are genetically tractable whereas many natural isoprenoid production pathways are prevalent in recalcitrant organisms that make high-titer production infeasible. Only recently have certain non-model organisms been engineered to yield comparable or higher isoprenoid titers than in E. coli and S. cerevisiae.

Despite the clear successes of isoprenoid production, E. coli and $S$. cerevisiae have significant disadvantages that limit successful bioproduction at scale. Precursor limitations, either the availability of IPP and DMAPP for direct synthesis of isoprenoids or the availability of MVA/MEP precursors, have been identified as a major obstacle to advancing isoprenoid synthesis ( $\mathrm{Zu}$ et al., 2020). Fine-tuning of metabolic pathways within the cell to balance cofactor supply by downregulation or upregulation of select enzymes has been identified as a major engineering opportunity and, although generally successful, often involves strain-specific and product-guided strategies ( $\mathrm{Zu}$ et al., 2020). Scaling of successful production is also limited by the necessity of episomal expression systems, which are ill-suited for industrial production due to the necessity of selective markers and their general instability.

A second major challenge in industrial isoprenoid biosynthesis is simultaneously increasing titer, rate, and yield while reducing the environmental and monetary burden intrinsic to industrial production. Precursor limitations are also complicated by isoprenoid production platforms that rely on sugar-based metabolism. Although sugars like glucose and glycerol provide high MVA/MEP flux by generation of G3P/pyruvate or acetyl-CoA, respectively, the high production costs are prohibitive to competition with petroleum-derived analogs. The greatest cost drivers of isoprenoid biosynthesis stem from carbon feed, which accounts for over $90 \%$ of production costs, and product yield (Wu and Maravelias, 2018).

A promising solution to exorbitant substrate costs is carbon source switching, especially to carbon waste streams like cellulosic biomass or $\mathrm{C} 1$ substrates (e.g., methane, methanol, carbon dioxide, and formate). Recent estimates assert that sugar switching from glucose to pretreated cellulosic biomass could yield a $53 \%$ decrease in cost (Wu and Maravelias, 2018) with further gains if organisms can simultaneously consume multiple substrates (e.g., hexose and pentose sugars). Growth and production on atypical carbon sources and native generation of secondary metabolites is prevalent among many microorganisms. Recent advances have capitalized on the diversity of microbial carbon assimilation pathways, especially in the elucidation of synthetic and natural C1 metabolic pathways that enable access to cheap, abundant carbon sources (Aldridge et al., 2021).

Many archaea have also evolved a robust array of resistance strategies to cope with inhibitory chemicals and conditions. These include tolerance mechanisms (efflux pumps, heat tolerance, membrane modifications, and general stress resistance; Dunlop, 2011) that facilitate extremophilic growth in inhospitable environments like anaerobic conditions or deep sea vents. These mechanisms in some cases directly involve secondary metabolite production and even enable enhanced tolerance to secondary metabolite toxicity (Dunlop et al., 2011). Toxicity tolerance is an appealing phenotype for biofuel production systems as well as for survival on substrates that are typically toxic to many microorganisms like pretreated lignocellulosic biomass (Dunlop et al., 2011). To date, bioproduction on pretreated cellulosic biomass has proven challenging due to the associated toxicity of the substrate, especially the prevalence of aromatic compounds. In response, researchers have begun focusing on resilient bioproduction chassis like Pseudomonas putida and Rhodosporidium toruloides that can readily degrade aromatic compounds (Yaegashi et al., 2017; Johnson et al., 2019).

Conversely, microbes with unique phenotypes tend to have limited metabolic toolkits available. Next generation sequencing technologies have expedited exploration and characterization of novel organisms from unique environments, yet direct 
engineering of such organisms for production remains a fundamental challenge. Neither $E$. coli or S. cerevisiae naturally accumulate isoprenoids at high titer and bioproduction is often limited to heavily modified strains with inducible episomal expression systems. Even so, the highest production of isoprenoids has been achieved in E. coli and S. cerevisiae (Moser and Pichler, 2019). As a result, there is a significant disparity between model and non-model isoprenoid production.

Addressing the disparity between the prevalence of nature's clever solutions to environmental challenges and the genetic tractability of those organisms remains a principal obstacle in isoprenoid bioproduction. In many instances, it poses the question of whether to heterologously express pathways in common metabolic chassis or to optimize pathways in situ, both of which come with drawbacks. In this review, we highlight recent advances in core understanding of isoprenoid synthesis, namely the elucidation of the archaeal MVA pathways, precursor flux modulation, and how those discoveries have contributed to novel isoprenoid production schemes. We then explore the exchange between lessons learned in the metabolic engineering of $E$. coli and S. cerevisiae and of non-model microorganisms with natural predispositions for atypical and economical carbon substrates. Pathways include $\mathrm{C} 1$ metabolism in methylotrophic organisms (Methanosarcina sp., Methylorubrum extorquens) and phototrophic microbes (cyanobacteria, purple non-sulfur bacteria, diatoms, and green algae) capable of fixing $\mathrm{CO}_{2}$. We also explore advances in engineering of oleaginous yeast naturally capable of efficient lipid toleration and accumulation ( $R$. toruloides and $Y$. lipolytica) and finally soil bacteria with special focus on their propensity for survival on and degradation of aromatic substrates (B. subtilis and P. putida). Collectively, these advances move isoprenoid biosynthesis toward economic and environmental feasibility.

\section{ADVANCES IN ISOPRENOID PATHWAY CONSTRUCTION:}

All isoprenoids are generated from the common cellular precursors acetyl-CoA or glyceraldehyde 3-phosphate (G3P) and pyruvate via either the MVA or MEP pathway, respectively (Figure 1). These pathways share no homology and are evolutionarily distinct. Comparisons of the MVA and MEP pathway efficiencies, cofactors, and energetic requirements have been well documented in previous reviews (Dugar and Stephanopoulos, 2011; Yadav et al., 2012). The recent characterization of archaeal MVA pathways, shunts, and alternative precursors for these pathways have harbored the development of unique and more efficient routes for isoprenoid production (Kang et al., 2017; Hayakawa et al., 2018; Clomburg et al., 2019).

\section{Mevalonate Pathway}

The MVA pathway is native to eukaryotes, some ancient and often predatory gram-positive bacteria (Pasternak et al., 2013), as well as, with some significant deviations, archaea (Boucher et al., 2004). The canonical MVA pathway commences with a Claisen condensation of two acetyl-CoA thioester molecules followed by five sequential enzymatic steps that ultimately yield IPP. IPP is then converted to DMAPP by the isopentenyl diphosphate isomerase (IDI) for further condensation into isoprenoid compounds.

Over the last decade, the origin of the archaeal MVA pathway - either progenating from horizontal gene transfer or a cenancestor - has been hotly debated. However, recent analysis of monophylogenetic candidate phyla radiation and DPANN (Diapherotrites, Parvarcheota, Aenigmarchaeota, Nanoarchaeota, and Nanohaloarchaeota) have provided conclusive evidence to support an extant ancestral MVA in all domains of life (Castelle and Banfield, 2018). Most notably, the archaeal pathway lacks PMK, PMD, and IDI1. Instead, archaea have an alternative IDI2 similar in function to IDI1 and rely upon the recently discovered isopentenyl phosphate kinase (Dellas et al., 2013) to generate IPP through unique MVA intermediates. Specifically, three distinctive archaeal MVA pathways have been elucidated: the haloarchaea-type MVA, the thermoplasma-type MVA, and the archaeal MVA pathway that is conserved throughout the kingdom (Hayakawa et al., 2018; Thomas et al., 2019; Yoshida et al., 2020) as depicted in Figure 1.

Beyond the perplexities of phylogenetic classification (hereditary, horizontal gene transfer, etc), the elucidation of archaeal MVA pathways and their associated enzymes has proven instrumental in optimizing $S$. cerevisiae/E. coli production titers by capitalizing upon enzyme promiscuity or efficiency. Collectively, heterologous expression and fine-tuning of the MVA pathway to minimize flux bottlenecks has included the expression of genes across different domains. Overexpression of HMGS and HMGR from Staphylococcus aureus (Tsuruta et al., 2009) as well as a kinase from the archaeal M. mazei (Primak et al., 2011), for example, was successfully shown to improve C5 isoprenoid accumulation and laid the groundwork for longer chain isoprenoid production via the MVA pathway (George et al., 2015).

\section{Methylerythritol Phosphate Pathway}

The MEP pathway is native to most Gram-negative bacteria and cyanobacteria as well as to algae and higher plants, but in the latter eukaryotes it is compartmentalized in the plastid. Despite being theoretically more efficient than the MVA pathway, the MEP pathway is more tightly regulated and challenging to engineer. Studies have elucidated rate limiting enzymatic steps in the MEP pathway, namely IDI and DXS, for $\beta$-carotene production (Yuan et al., 2006). However, overexpression of MEP pathway genes can also have deleterious effects on actual isoprenoid synthesis due to accumulation of intermediates. Our fundamental understanding of MEP pathway regulation is incomplete, encompassing some feedback and feedforward mechanisms between downstream isoprenoids and MEP intermediates (Bitok and Meyers, 2012). Studies in higher plants and algae, which have demonstrated that circadian light/dark cycling have a significant influence on pathway regulation, further complicate our understanding (Vranová et al., 2012). 


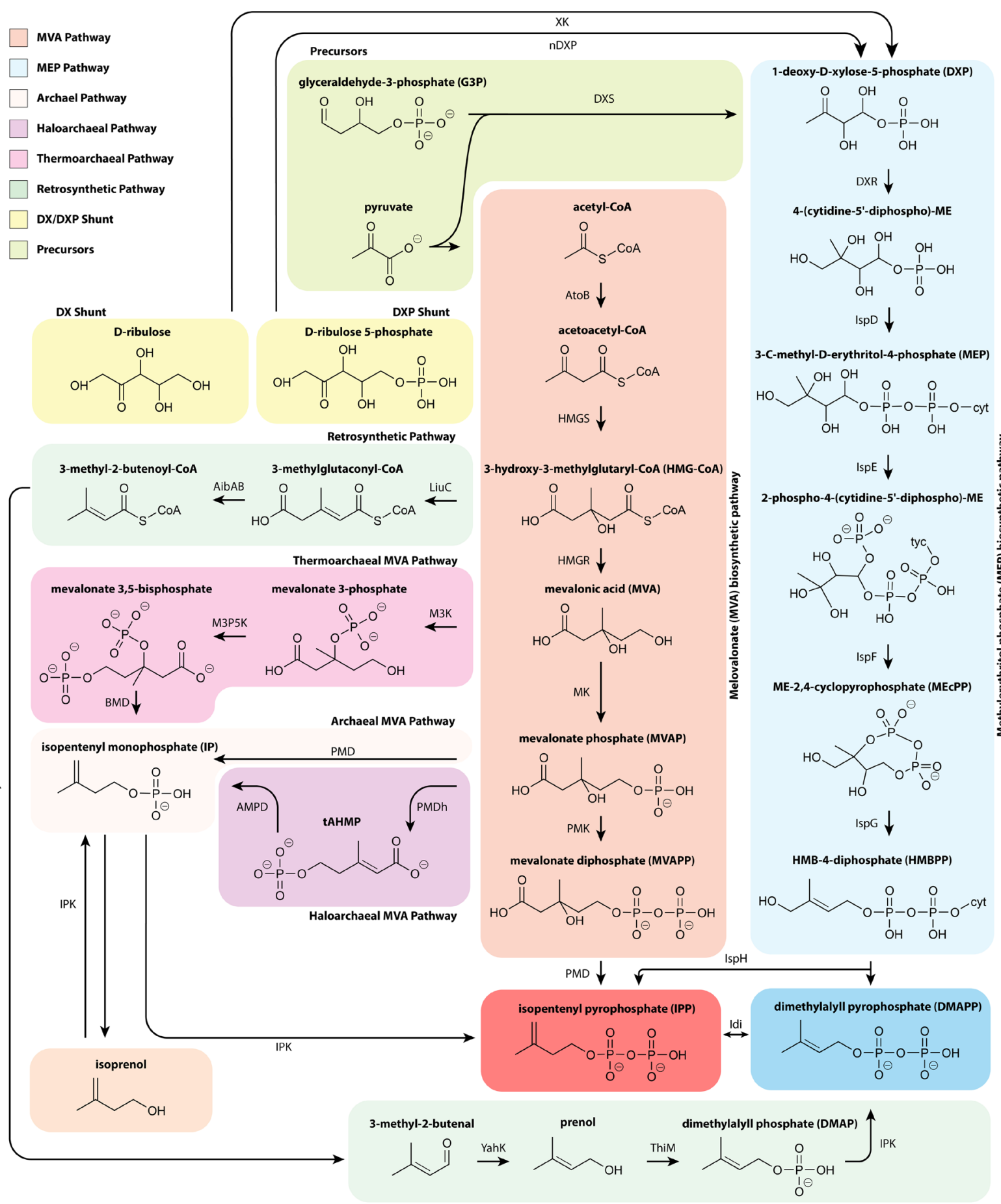

FIGURE 1 | A depiction of isoprenoid synthesis through the core 6 enzyme MVA and 7 enzyme MEP pathways. Also depicted are the newly discovered archaeal branches from the MVA pathway. The thermoarchaeal-type branch begins with mevalonic acid whereas the archaeal and haloarchaeal-type branches stem from MVAP. Typically, isoprenoids are synthesized by acetyl-CoA, pyruvate, and G3P, however IPP and DMAPP can also be synthesized from C5 alcohols, D-ribulose or D-ribulose-5-phosphate, and a synthetic route in which HMG-CoA is ultimately converted to prenol. AibAB, 3-methylglutaconyl-coenzyme decarboxylase; AtoB, acetyl-CoA acetyltransferase; BMD, bisphosphomevalonate decarboxylase; cbjALD, 3-methylcrotonyl-CoA reductase; DXR, 1-deoxy-D-xylulose 5-phosphate reductase; DXS, 1-deoxy-D-xylulose 5-phosphate synthase; HMGR, 3-hydroxy-3-methylglutaryl-CoA reductase; HMGS, 3-hydroxy-3-methylglutaryl-CoA synthase; IDI, isopentenyl diphosphate isomerase; IPK, isopentenyl phosphate kinase; IspD, 2-C-methyl-D-erythritol 4-phosphate cytidylyltransferase; IspE, 4-diphosphocytidyl-2-C-methyl-D-erythritol kinase; IspF, 2-C-methyl-D-erythritol 2,4-cyclodiphosphate synthase; IspG, 4-hydroxy-3-methylbut-2-enyl diphosphate synthase; IspH, 4-hydroxy-3-methylbut-2-enyl diphosphate reductase; LiuC, 3-hydroxy-3-methylglutaryl CoA dehydratase; M3K, Mevalonate 3-kinase; M3P5K, Mevalonate 2-phosphate-kinase; nDXP, 1-deoxyxylulose-5-phosphate synthase; PMD, phosphomevalonate decarboxylase; PMK, phosphomevalonate kinase; tAHMP, anhydromevalonate diphosphate decarboxylase; ThiM, hydroxyethylthiazole kinase; XK, xylulose kinase; YahK, aldehyde reductase. 
A recent metabolic control analysis employed -omics studies with recombineering to show that, normalized to DXS flux, IspG is the rate limiting step for isoprene synthesis with other enzymes increasing linearly with DXS concentration (Volke et al., 2019). This is an important finding as isoprene is the simplest hemiterpene and therefore a good reporter for MEP tuning. Yet production did not increase with overexpression of IspG and IspH, suggesting instead that other cofactors may be limiting (Volke et al., 2019). Indeed, careful balancing of IspG and IspH expression has shown enhanced $\beta$-carotene and $\alpha$-lycopene production (Li et al., 2017), suggesting that pathway tuning should be based on an intricate, product-driven approach (e.g., different tuning for isoprene vs. higher chain length terpenoids) rather than an intuitive, generalizable rule. In general, careful expression balancing has been the most successful strategy to MEP pathway optimization due to the complexity of regulatory mechanisms in E. coli, though even careful balancing in other organisms like the cyanobacterium Synechococcus elongatus has proven challenging (Englund et al., 2018).

\section{Synthetic Isoprenoid Production Pathways}

Although essential to isoprenoid production, high IPP and DMAPP accumulation is toxic and can result in significant growth inhibition (George et al., 2018). This dilemma has led to a number of clever strategies for synthetic "growthdecoupled" and "bypass" isoprenoid production routes that comprise components of MVA/MEP pathways but avoid IPP/ DMAPP accumulation. Many of these strategies have been informed or directly use elements from the recently elucidated archaeal MVA pathways, either through direct codon optimized expression or as templates for engineering promiscuous activity. A mevalonate decarboxylase from Halobacterium volcanii, for example, was expected to demonstrate conversion of MVAP to IP and employed as a template to rationally design PMDs for C5 alcohol production. The strategy successfully enhanced isoprenol production by bypassing intracellular IPP accumulation (Vannice et al., 2014; Kang et al., 2016). Further mutagenesis of a S. cerevisiae PMD in tandem with an endogenous phosphokinase resulted in an IPP-bypass pathway that yields IP and ultimately the highest isoprenol titer reported at $10.8 \mathrm{~g} / \mathrm{L}$ (Kang et al., 2017, 2019).

Retro-biosynthetic approaches postulated that archaeal IPKs could enable phosphorylation of the C5 alcohols (isoprenol and prenol) into IPP and DMAPP, respectively. Direct feeding of alcohols for production of isoprenoid precursors could thereby decouple isoprenoid production from central carbon metabolism. Of particular interest were IPKs from Halobacterium volcanii, Methanothermobacter thermautotrophicus, Thermoplasma acidophilum, and Methanocaldococcus jannaschii (Chatzivasileiou et al., 2019). In one study, IPKs from the latter three archaea were screened for activity and cloned into an E. coli strain harboring a $\beta$-carotene production pathway (Liu et al., 2016). Expression of the IPK from T. acidophilum and feeding of $2 \mathrm{mM}$ prenol resulted in a $45 \%$ increase in $\beta$-carotene production and was further improved by site-specific mutagenesis to $97 \%$ (Liu et al., 2016). Growth-decoupled production of lycopene was also demonstrated by overexpressing a codon-optimized T. acidophilum IPK paired with an endogenous $E$. coli phosphatase PhoN, with titers nearing $190 \mathrm{mg} / \mathrm{L}$ in an mixture of $2.5 \mathrm{mM}$ prenol and isoprenol (Chatzivasileiou et al., 2019; Clomburg et al., 2019; Lund et al., 2019). IPK-mediated production of carotenoid and neurosporene was also improved in E. coli by 18 -fold and 45 -fold, respectively, by decoupling terpene synthesis from central carbon metabolism through production on C5 alcohols (Rico et al., 2019).

Several other strategies utilize two upper MVA pathway genes (E. coli AtoB, Staphylococcus aureus HMGS) prior to diverging with the expression of the hydratase LiuC and Myxococcus xanthus decarboxylase AibAB (Clomburg et al., 2019; Eiben et al., 2020). From there, Eiben et al. demonstrated $80 \mathrm{mg} / \mathrm{L}$ isopentanol production through subsequent expression of $M$. xanthus AibC and Clostridium acetobutylicum AdhE2 (Eiben et al., 2020). In a more holistic approach, AibAB was followed with expression of the Clostridium beijerinckii acyl-CoA reductase (cbjALD), and E. coli YahK to generate prenol at the highest titer reported for biological production (Figure 1; Clomburg et al., 2019). Conversion of prenol to DMAP was then accomplished by the E. coli hydroxyethylthiazole kinase (ThiM) and finally converted to DMAPP by the M. thermoautotrophicus IPK (Clomburg et al., 2019). The unique approach employed by Clomburg et al. succinctly demonstrates how novel enzymes wrought by recent discoveries can be instrumental in designing pathways that circumvent metabolic bottlenecks to yield high titer production platforms.

To conclude, several studies have explored novel precursors to the MEP pathway using ribulose. The initial step in the MEP pathway, condensation of G3P and pyruvate with DXS, results in production of DXP with the loss of a $\mathrm{CO}_{2}$ molecule or one sixth of total carbon (Kirby et al., 2015). A D-ribulose 5-phosphate shunt by $\mathrm{nDXP}$ was initially explored in $E$. coli by a semi-rational approach, which identified yajO and ribB gene mutants as candidate enzymes and improved carbon efficiency by direct conversion of C5 sugars to C5 MEP intermediates. Expression of the nDXP shunt enabled a 4-fold increase in MEP derived bisabolene production (Kirby et al., 2015). This approach was further demonstrated in $P$. putida by expression of the mutant ribB gene, but with low efficiency (Hernandez-Arranz et al., 2019). In an analogous work, promiscuous activity of fructose-6-phosphate aldolase in $E$. coli was used to generate D-ribulose from the glycolaldehyde and hydroxyacetone. Another DXP shunt overexpressed a native xylulose kinase (King et al., 2017). These novel shunts, like the archaeal informed MVA pathways, have the potential to alleviate precursor flux limitations. While the MVA pathway modifications have had clear success, it has yet to be determined whether these shunts can address the regulatory challenges associated with MEP derived isoprenoid production. 


\section{ADVANCES IN ISOPRENOID FUNCTIONALIZATION}

The C5 precursors IPP and DMAPP are dephosphorylated, cyclized, and modified to create a structurally diverse group of over 70,000 chemicals through a coordinated enzyme network (Kirby and Keasling, 2009; Moser and Pichler, 2019). The first stage or module of isoprenoid biosynthesis is characterized by the successive addition of the diphosphate precursor via headto-head or tail-to-head condensation. The second module is an operation or series of operations conducted by terpene synthases (TSs) in which the terpenoid skeleton is dephosphorylated and cyclized. The third module involves further decoration by cytochrome P450s (CYPs), acetyltransferases, methyltransferases, dehydrogenases, and in some cases, glycosylations. This overall framework is consistently repeated in nature with some variations (Zhou and Pichersky, 2020). In this section we discuss recent advances in the functionalization of isoprenoids. Broad ranges of chemical production targets have been demonstrated and scaled from biofuels to pharmaceuticals by heterologous expression of prenyl diphosphate synthases, TSs, and CYPs.

\section{Cytochrome P450s}

Heme-thiolate monooxygenases or CYPs are an interesting class of enzymes that functionalize terpenes through oxygenation reactions (hydroxylation, dealkylation, demethylation, decarboxylation, cyclization, C-C bond cleavage, among others) and present an important opportunity for generating highly decorated natural products. To date, over 300,000 CYPs have been discovered, with less than $1 \%$ actually characterized ( $\mathrm{Li}$ et al., 2020; Liu et al., 2020). There is particular interest to produce CYP-derived terpenoids in microbial chassis due to the high barriers of slow growth and costly deconstruction inherent to native plant extraction.

Engineering CYPs has significant implications for novel and unnatural bioproducts (Helfrich et al., 2019; Xiao et al., 2019). The range of oxygenated terpenes is complemented by the sheer expanse of CYP availability in plants (Zhou and Pichersky, 2020). For example, CYPs are critical for the production of bioactive molecules with high pharmacological impacts. Case studies of microbial expression include production of precursor intermediates to artemisinin (CYP71AV1, aaCPR) and taxadiene (CYP725A4, tcCPR), which are natively produced by wormwood (Artemisia annua) and the Pacific yew tree (Taxus brevifolia) and were heterologously expressed in E. coli and S. cerevisiae, respectively. Indeed, the diversity and complexity of plant TSs, presented elsewhere (Karunanithi and Zerbe, 2019) offer tremendous potential as candidates for microbial production.

These specific examples demonstrate successful engineering of CYPs for pharmaceutical production, however functional plant CYP expression in microbes has proved challenging. Unfortunately, E. coli cannot naturally perform most posttranslational modifications and expression of membrane bound proteins like CYPs generates inclusion bodies or aggregates of insoluble proteins. The production of oxygenated taxanes in E. coli (Biggs et al., 2016), for example, required extensive engineering of the CYP redox-partner cytochrome P450 reductase $(\mathrm{CPR})$ pairings, $\mathrm{N}$-terminal modifications for better solubility, and had significant repercussions on upstream MEP pathway balance. These pairings and modifications are necessary for any heterologous CYP expression in E. coli and vary depending on the selected proteins. Remarkably, although yeasts are naturally capable of many posttranslational modifications, express native CPRs, and require less $\mathrm{N}$-terminal modification, meta-analyses have shown that E. coli studies tend to have higher yield CYPs than $S$. cerevisiae despite the necessity of many more genetic modifications (Hausjell et al., 2018). Many CYP-reductase pairings have been explored in E. coli and S. cerevisiae as listed in Table 1.

In recent years, microbial expression of CYPs has produced many variable length terpenoids (Figure 2). Of special significance are the monoterpene perillyl alcohol, an anti-cancer drug, from limonene (Alonso-Gutierrez et al., 2013) and the sesquiterpenes nootkatone, a pharmaceutical, from valencene (Guo et al., 2018) and zerumbone, an antioxidant, from $\alpha$-humulene (Zhang et al., 2018). Of further interest are the pharmacologically relevant diterpenes taxadiene and oxygenated casbenes as well as triterpenoids glycerrhetol from 11-oxo-b-amyrin and betulinic acid from lupeol.

There have been many breakthroughs in CYP-derived terpenes in the last 5 years that may provide guidance in the engineering of other CYP expression systems. Their interest has led to toolkits for enhanced CYP selection for targeted product engineering to streamline oxyfunctionalization of terpenes (Hernandez-Ortega et al., 2018). Challenges remain with regards to CYP promiscuity, enantiomeric purity, and, perhaps most importantly, production titers. Hopefully, such tools will guide hypotheses and penetrate into the library of known but currently inaccessible plant bioactive terpenes for use in high titer therapeutic production.

\section{Atypical Terpenoid Production}

Several studies have investigated novel isoprenoid chain lengths through the expression of unique S-adenosylmethionine (SAM)-dependent methyltransferases that methylate the fundamental IPP/DMAPP building blocks and thereby break the natural C5 dogma. In a pioneering study, a GPP methyltransferase (2meGPP) from the cyanobacterium Pseudanabaena limnetica was expressed in S. cerevisiae with its native 2-methylisoborneol synthase and then with seven distinct plant monoterpene synthases (Ignea et al., 2018). Each synthase generated a unique fingerprint of novel C11 compounds. By demonstrating a range of $\mathrm{C} 11$ targets, the authors provided a proof of concept for future enzyme optimization strategies toward specific C11 targets. In another work, IPP SAM-dependent methyltransferases enable conversion of IPP to C6 and C7 prenyl diphosphates with a methyltransferase from Streptomyces monomycini, which could then generate $\mathrm{C} 11, \mathrm{C} 16$, and $\mathrm{C} 17$ terpenes as well 
TABLE 1 | CYP expression, reductase pairing, and production of oxygenated terpenoids in various microbial hosts.

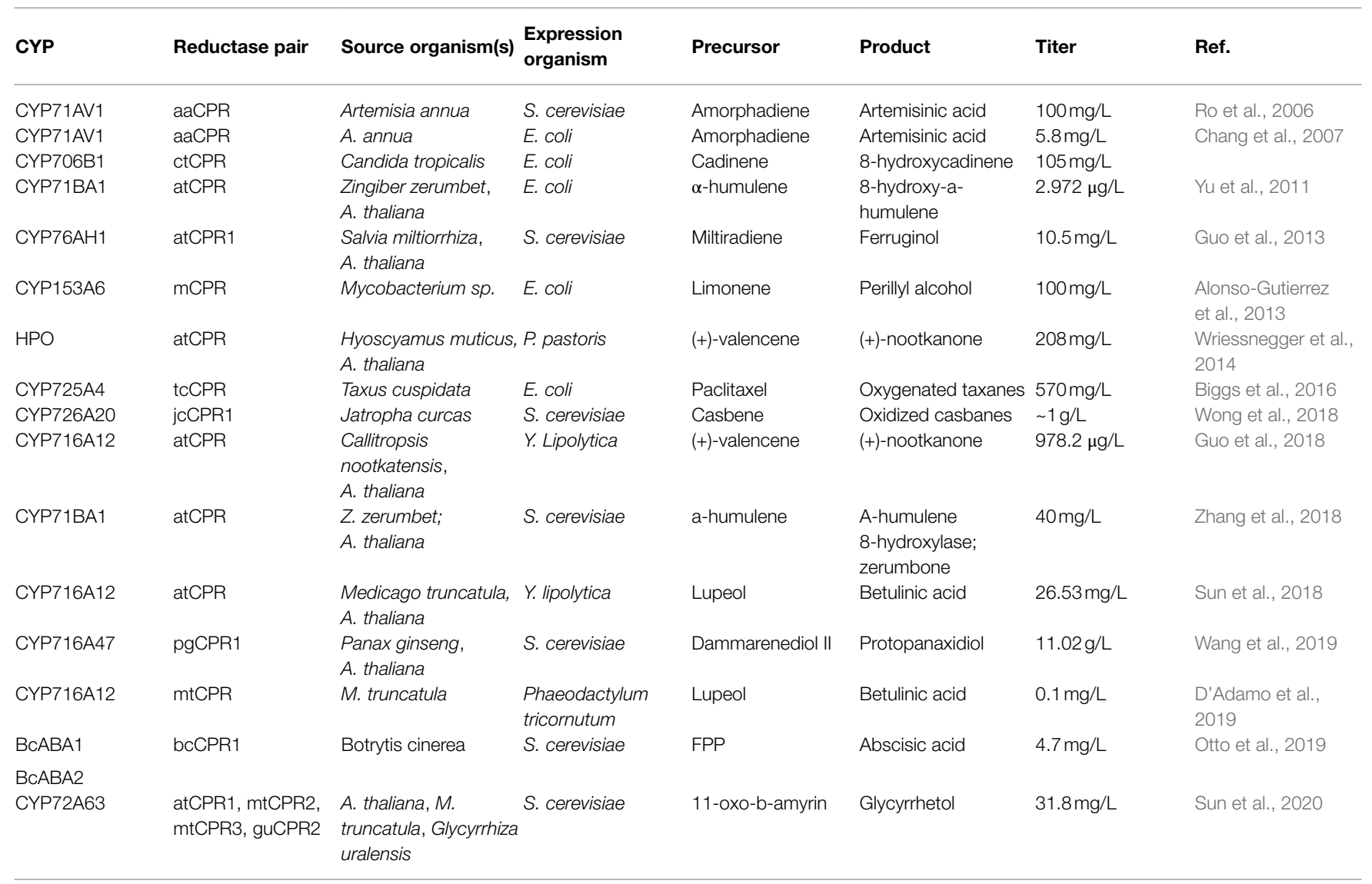

Culture conditions, scale, and medium vary significantly. HPO, hyoscyamus muticus premnaspirodiene oxygenase; CPR, cytochrome P450 reductase.

as zeaxanthin-like C41, C42, and C43 compounds depending on methylation (Drummond et al., 2019). A product-driven approach was also able to elucidate a C-methyltransferase that generated sodoferin, an atypical C16 sesquiterpene (von Reuss et al., 2018). The work demonstrated that sodoferin, which is produced naturally and perhaps exclusively by a Serratia plymuthica, could be produced in E. coli through heterologous expression of the methyltransferase and TS (von Reuss et al., 2018). A final, completely divergent approach utilizes a thiolase from lepidoptera (butterflies and moths) that naturally produces juvenile hormones in the form of C16 methylated diterpenoids (Eiben et al., 2019). Specifically, the thiolase $\mathrm{PhaA}$ condenses a propionyl-CoA with an acetyl-CoA as opposed to the standard AtoB of the MVA pathway, which condenses two acetyl-CoA substrates.

It is probable that the control of specific methylation sites decreases with compound size such that targeting specific triterpenoids would remain an obstacle. In the case of C11 targets, site-directed mutagenesis of the monoterpene synthases did enable higher selectivity (Ignea et al., 2018), which is encouraging for future engineering. These unique approaches have expanded the boundaries of isoprenoid synthesis well beyond the $\mathrm{C} 5$ rule, though admittedly practical applications of these novel compounds have yet to be realized.

\section{Meroterpenoids}

Partial isoprenoids or meroterpenoids are a class of compounds containing an isoprenoid chain paired with another structure and may have beneficial bioactive properties. Broadly, meroterpenoids include cytokinins, quinones, steroids, and porphyrins like heme $\mathrm{A}$ and chlorophyll a. The optimization of heterologous meroterpenoids poses a unique engineering challenge as the isoprenoid compound must be cogenerated with another structure, then converted to the terpenoid by a specified synthase.

A good case study is the production of cannabinoids in S. cerevisiae. Cannabinoids are of commercial interest but, like many natural products, suffer from low in planta yields. In a recent publication, the production of olivetolic acid from acetyl-CoA was engineered using a six gene pathway (Luo et al., 2019). Prenylation of olivetolic acid using a Cannabis sativa prenyltransferase (csPT4) and further heterologous synthases led to in vivo production of cannabigerolic acid, $\Delta$ 9-tetrahydrocannabinolic acid, cannabidiolic acid, $\Delta$ 9-tetrahydrocannabivarinic acid, and cannabidivarinic acid (Luo et al., 2019). The work not only presents a novel production scheme in $S$. cerevisiae but demonstrates the ease with which transgenic elements can be translated into production chassis. 


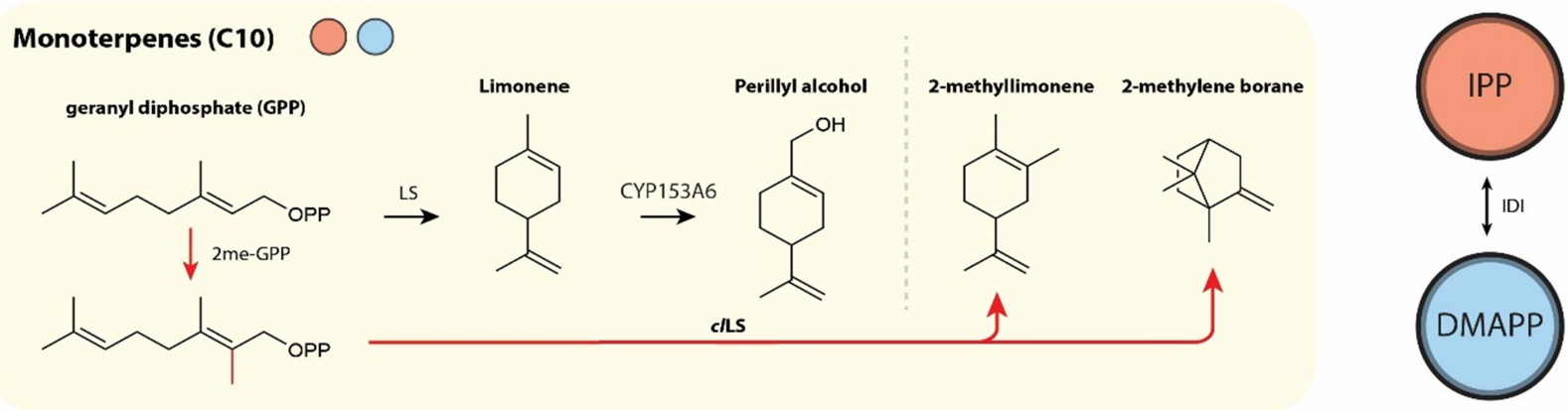

\section{Sesquiterpenes (C15) $\bigcirc \bigcirc \bigcirc$}
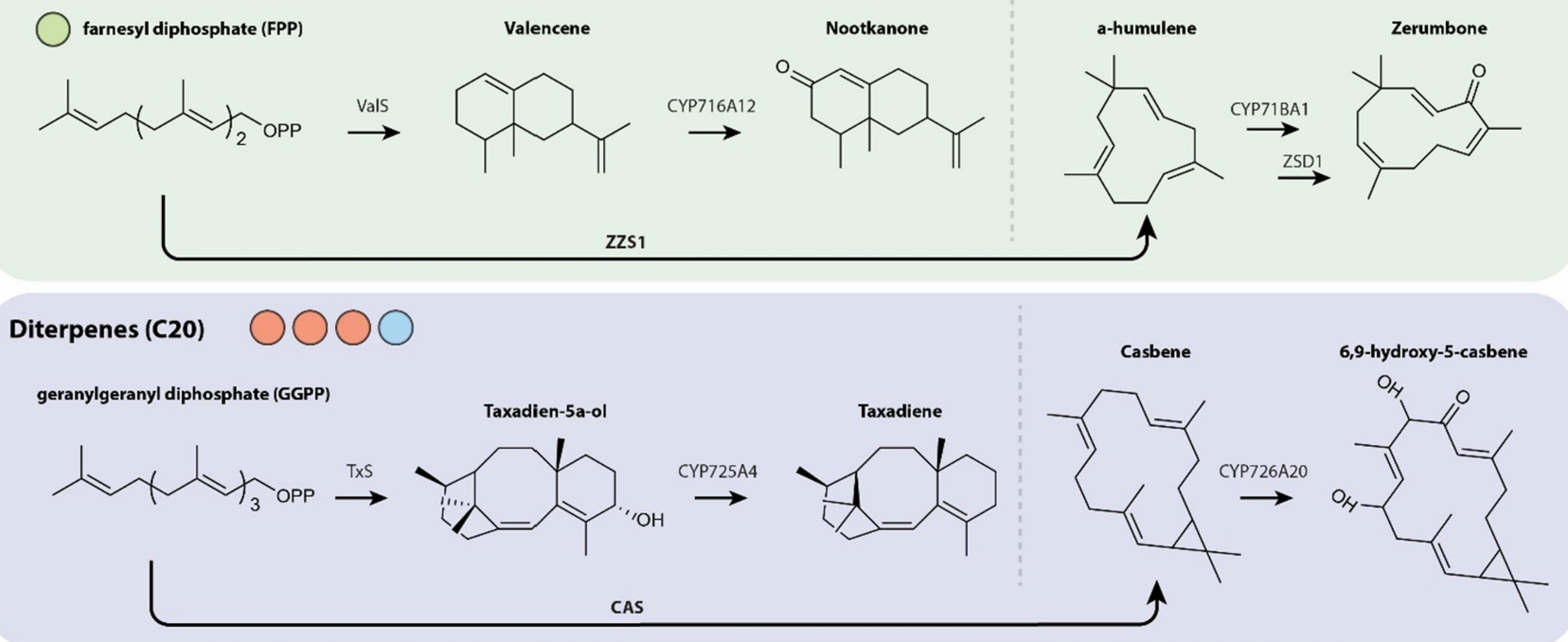

\section{Triterpenoids (C30) $\bigcirc \bigcirc \bigcirc \bigcirc \bigcirc \bigcirc$}
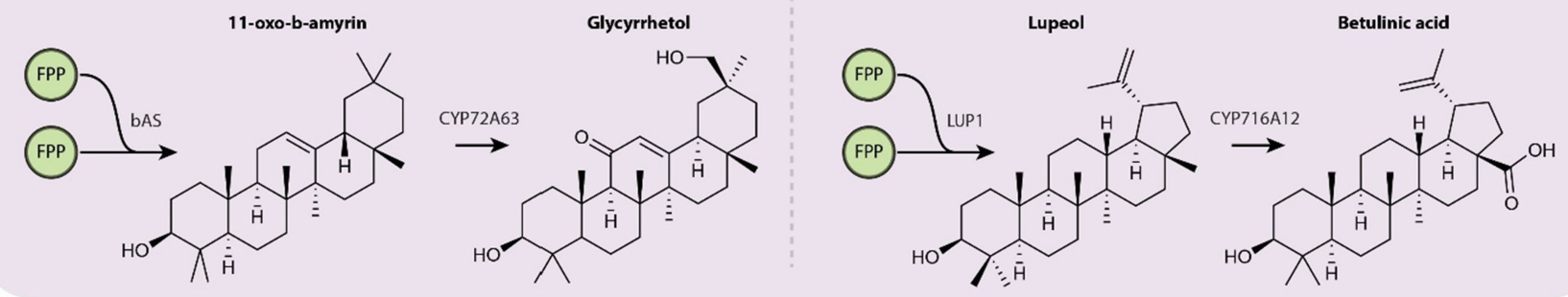

FIGURE 2 | Overview of varied length terpenoids from their diphosphate precursors (red circle, IPP; blue circle, DMAPP; green circle, FPP) with further modification by TSs and subsequent decoration by CYPs. The production of unnatural C11 monoterpenoid compounds via methyltransferase 2me-GPP is indicated with red arrows. CAS, casbene synthase; LS, limonene synthase; c/LS, Citrus limon limonene synthase; 2me-GPP, GPP methyltransferase; TxS, taxadiene synthase; bAS, $\beta$-amyrin synthase; LUP1, lupeol synthase 1; ValS, valencene synthase; ZSD1, zerumbone synthase; ZZS1, $\alpha$-humulene synthase.

Another relevant example is the production of prenylated flavonoids, which are derived from cyclic amino acid biosynthesis and can serve as nutraceuticals and medicines. They, again, are found in low natural abundance in plant species like Sophora flavescens, a shrub and Humulus lupulus, brewing hops (Yang et al., 2015). Production of naringenin in S. cerevisiae coupled with overexpression of a plant flavonoid prenyltransferase enabled production of the pharmaceutically relevant 8-prenylnaringenin (Levisson et al., 2019). Although both strategies were limited to yeast, they underline the flexibility of engineering meroterpenoid production in microbes to address commercial needs and provide a promising opportunity for accessing low abundance natural products.

Unique meroterpenoids are also generated in high natural abundance in certain microbes. Archaea differ from bacteria primarily in membrane composition. Archaea produce isoprenoid-derived glycerol lipid ethers (namely ester linked sn-glycerol 3-phosphates rather than ether linked sn-glycerol 1-phosphates) that facilitate growth in unique environments. Full reduction of these long length C20/C40 membrane isoprenoid 
chains is accomplished by downstream geranylgeranyl reductases (GGR). While they provide an evolutionary advantage for survival in extreme conditions, membrane isoprenoids may also be utilized to generate unsaturated chemicals of interest (Jain et al., 2014). Archaeal lipids, namely archaeol and caldarchaeol, have been identified as potentially valuable for the formation of archaeosomes. Archaeosomes are lipid vesicles composed of archaeal derived lipids and have shown higher physicochemical stability than liposomes, a conventional drug delivery system. As a result, archaeosomes have been singled out as a possible adjuvant and could prove particularly valuable in slow release drug delivery systems (Caforio and Driessen, 2017).

\section{INDUSTRIAL PRODUCTION FROM C1 CHEMICAL FEEDSTOCKS}

C1 substrates are typically generated as industrial and petrochemical byproducts and, in general, C1 substrates are stable, abundant, and inexpensive. Advances in sequestration and hydrogenation of atmospheric $\mathrm{CO}_{2}$ via heterogeneous catalysts have enabled the conversion of emissions into valuable $\mathrm{C}_{2+}$ substrates (Ye et al., 2019). Metabolic engineering of organisms capable of $\mathrm{C} 1$ growth is an enticing opportunity for achieving cost parity with petrochemical products while simultaneously improving sustainability metrics. C1 metabolism may be subdivided into phototrophic, methylotrophic, or formatotrophic microbes that consume $\mathrm{CO}_{2}$, methane/methanol, and formate/formic acid, respectively. Here, we describe recent approaches for converting $\mathrm{C} 1$ substrates into isoprenoid precursors with specific attention to works demonstrating isoprenoid production. The generalized pathways for C1 metabolism are illustrated in Figure 3.

\section{Methylotrophic Isoprenoid Production}

Methane is an abundant byproduct of many chemical processes including fracking and petroleum drilling (Clomburg et al., 2017). In terms of greenhouse gas emissions, methane is approximately 20 times more potent than $\mathrm{CO}_{2}$ and excess capacity is flared at refineries, lending to an increase in direct $\mathrm{CO}_{2}$ emissions and a loss of revenue (Conrado and Gonzalez, 2014). Methane is also generated through anaerobic digestion of waste biomass. To date, many successful chemical platforms utilize methane as a feedstock to generate alcohols, carboxylic acids, as well as other common $\mathrm{C} 2 / \mathrm{C} 3$ chemicals (Kuhl et al., 2012). While biological conversion rates tend to be lower, certain methylotrophic organisms have arisen as potential candidates to capitalize upon methane/methanol availability for more complex bioproduction. In this section, we discuss recent advances in isoprenoid biosynthesis in methylotrophic cell factories across domains.

Archaea are well regarded for their ability to thrive in nutrient-limited anaerobic and extreme conditions. As a result, many archaea have evolved highly efficient strategies for $\mathrm{C} 1$ assimilation. On one hand this has helped elucidate more efficient MVA pathways as previously described, but on the other it makes the engineering of tightly regulated archaeal pathways that are geared toward energy conservation thermodynamically challenging. Nonetheless, one study demonstrated production of isoprene from methanol in Methanosarcina acetivorans and Methanosarcina barkeri under anaerobic conditions and showed a redirection of electron flux from membrane precursors in favor of isoprene accumulation (Aldridge et al., 2021). The diverted isoprene accounted for $4 \%$ of total carbon flux.

More substantial success has been achieved in methylotrophic bacteria, many of which thrive in more mesophilic conditions. Methylotrophic bacteria are predominantly divided into two types: Type I assimilates formaldehyde using the RuMP cycle and Type II assimilates formaldehyde via the serine cycle. Methylorubrum extorquens AM1 (formerly Methylobacterium extorquens AM1), a Type II methylotroph, has been studied for over 60 years such that many genetic tools are available (-omics data, metabolic networks, genome-scale model; Schrader et al., 2009). M. extorquens fermentations have produced methanol-derived products ranging from 1-butanol ( $\mathrm{Hu}$ and Lidstrom, 2014) to polymers (Orita et al., 2014). A series of stepwise optimizations in $M$. extorquens AM1 included heterologous expression of the M. xanthus MVA genes, an FPP synthase from $S$. cerevisiae, $\alpha$-humulene synthase from Zingiber zerumbet, and reduced carotenoid flux. Combinedly, these modifications resulted in the accumulation of $1.65 \mathrm{~g} / \mathrm{L}$ $\alpha$-humulene on methanol in fed-batch cultivation, which stands as the highest titer reported (Sonntag et al., 2015). Other works have explored high titer production of the MVA pathway intermediates, including $2.59 \mathrm{~g} / \mathrm{L}$ mevalonic acid from methanol on minimal media using a mevalonate biosensor strategy (Liang et al., 2017), which suggests that high titer production of other isoprenoids is also possible. As another attractive feature, $M$. extorquens harbors the ethylmalonyl-CoA pathway (EMCP) that includes a series of anaperlotic activated CoA esters useful for pathway remodeling (Schrader et al., 2009; Schada von Borzyskowski et al., 2018). Interpathway metabolite exchange has risen as a major avenue for further $\mathrm{C} 1$ metabolism, especially for formatotrophs as discussed later.

Recently, the methylotrophic yeast Pichia pastoris (formerly known as Komagataella phaffii) has arisen as a promising candidate for isoprenoid production on methanol. P. pastoris maintains several unique characteristics including a tightly regulated and highly expressed alcohol oxidase AOX1, which catalyzes oxidation of methanol to formaldehyde. AOX1 is strongly induced by methanol but repressed by glucose and glycerol (Hartner and Glieder, 2006). As a result, fed-batch production schemes have been designed to partition high cell density growth on glycerol/glucose and production on methanol. This strategy has successfully demonstrated the production of $714 \mathrm{mg} / \mathrm{L}$ lycopene (Zhang et al., 2020), (+)-ambrein, squalene (Moser et al., 2018), and $208 \mathrm{mg} / \mathrm{L}$ (+)-nootkatone (Wriessnegger et al., 2014). Interestingly, the latter study successfully used an approach for CYP production that had failed in S. cerevisiae. More involved engineering of $P$. pastoris has demonstrated de novo production using heterologously expressed 

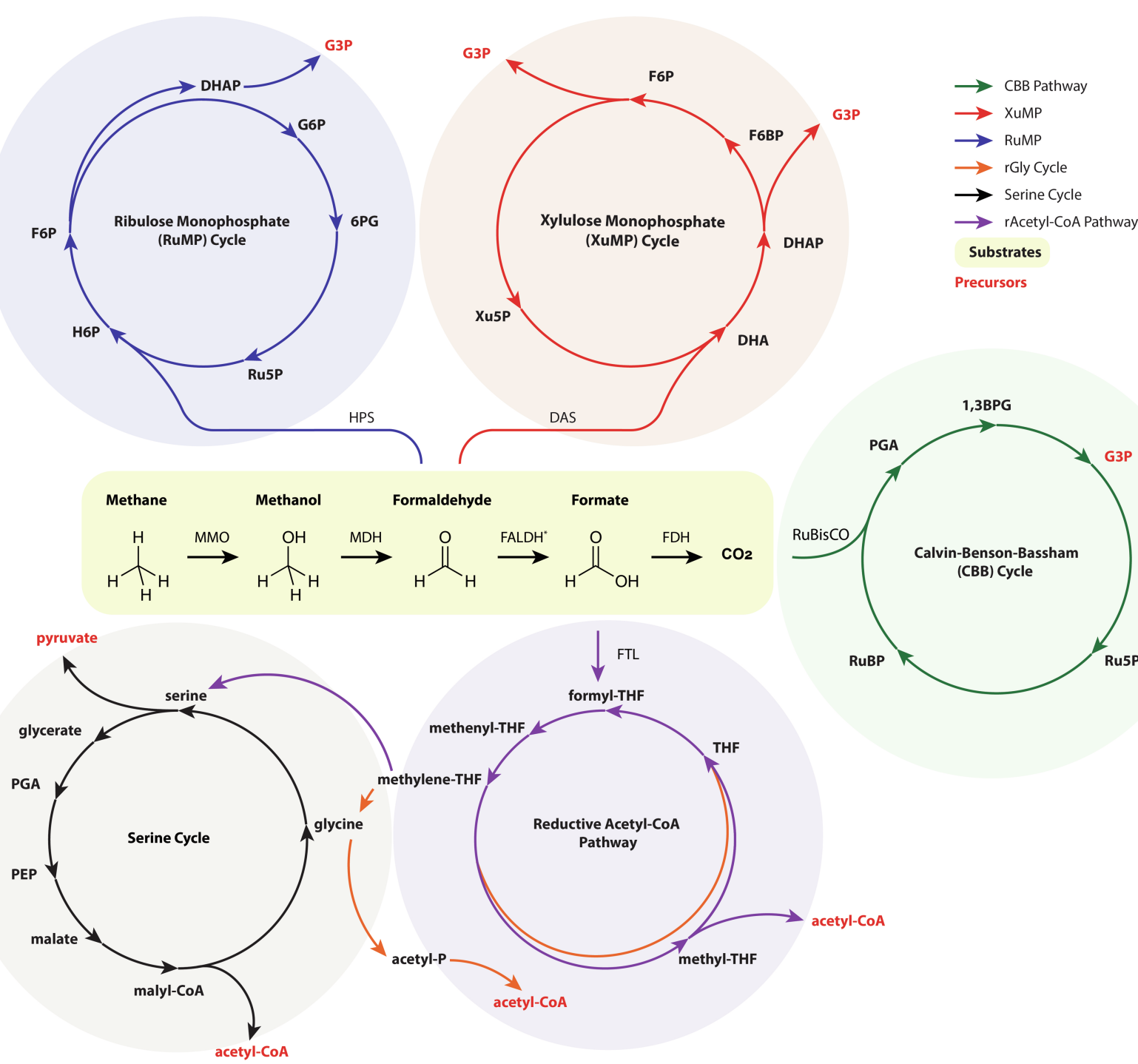

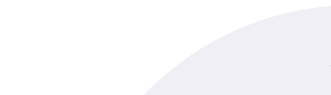
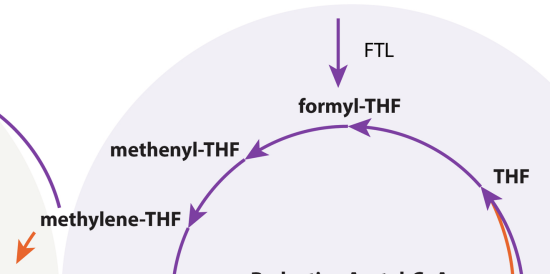
(a)

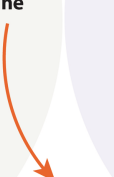

Reductive Acetyl-CoA Pathway

FIGURE 3 | An amalgamated and simplified depiction of relevant C1 metabolic pathways, namely the ribulose monophosphate (RuMP) cycle, the xylulose monophosphate (XuMP) cycle, the Calvin-Benson-Bassham (CBB) cycle, the serine cycle, reductive acetyl-CoA (Wood-Ljungdahl) cycle, and the reductive glycine pathway. Intracycle reactions and conversion of metabolites by glycolysis is not shown. Emphasis is placed on precursors for isoprenoid and central carbon metabolism, namely G3P, acetyl-CoA, and pyruvate. For clarity, only the enzymes involved in the initial C1 assimilation are listed. For simplicity, FALDH is the depicted enzyme for conversion of formaldehyde to formate and the canonical methanogenic reactions are omitted. 1,3BPG, 1,3-bisphosphoglyceric acid; 6PG, 6-phosphogluconate; DAS, dihydroxyacetone synthase; DHA, dihydroxyacetone; DHAP, dihydroxyacetone phosphate; F6BP, fructose 6-bisphosphate; F6P, fructose 6-phosphate; FALDH, formaldehyde dehydrogenase; FDH, formate dehydrogenase; FTL, formate-THF ligase; G3P, glyceraldehyde 3-phosphate; G6P, glucose 6-phosphate; H6P, hexulose 6-phosphate; HPS, hexulose-6-phosphate synthase; MDH, methanol dehydrogenase; MMO, methane monooxygenase; PEP, phosphoenolpyruvate; PGA, 3-phosphoglyceric acid; Ru5P, ribulose 5-phosphate; RuBisCO, ribulose 1,5-bisphosphate carboxylase-oxygenase; RuBP, ribulose bisphosphate; THF, tetrahydrofolate; Xu5P, xylulose 5-phosphate.

Calvin-Benson-Bassham (CBB) Cycle enzymes along with native genes in the xylulose monophosphate (XuMP) cycle and deletion of certain fatty acid enzymes, ultimately yielding a mutant exhibiting autotrophic growth on $\mathrm{CO}_{2}$ (Gassler et al., 2020).

Methylotrophic production has garnered special interest for reducing bioproduction costs either through valorization of commercial waste streams or $\mathrm{CO}_{2}$ conversion. Table 2 provides a list of methylotrophic production strains, their respective C1 substrates, and product titers. In most cases, titers are significantly lower than comparative production in E. coli or $S$. cerevisiae and methylotrophic cultures require significant supplementation with a rich medium that may somewhat reduce the benefits of $\mathrm{C} 1$ production.

Past attempts at methylotrophic production have historically been hindered by low carbon and energy efficiencies and addressing these issues by leveraging RuMP/Serine cycles with the $\mathrm{CBB}$ cycle have been long postulated (Conrado and Gonzalez, 2014). Recently, major breakthroughs in P. pastoris demonstrated 
TABLE 2 | Production of isoprenoids by methylotrophic organisms.

\begin{tabular}{|c|c|c|c|c|}
\hline Organism & Feedstock & Product & Titer & Ref. \\
\hline $\begin{array}{l}\text { Methylomonas } \\
\text { sp. } 16 a\end{array}$ & Methane & astaxanthin & $2.4 \mathrm{mg} / \mathrm{g}$ CDW & Ye et al., 2007 \\
\hline P. pastoris & Methanol** & (+)-nootkanone & $208 \mathrm{mg} / \mathrm{L}$ & $\begin{array}{l}\text { Wriessnegger } \\
\text { et al., } 2014\end{array}$ \\
\hline P. pastoris & Methanol** & (+)-valencene & $136 \mathrm{mg} / \mathrm{L}$ & \\
\hline M. extorquens & Methanol & a-humulene & $1.65 \mathrm{~g} / \mathrm{L}$ & $\begin{array}{l}\text { Sonntag et al., } \\
2015\end{array}$ \\
\hline P. pastoris & Methanol** & (+)-ambrein & $105 \mathrm{mg} / \mathrm{L}$ & $\begin{array}{l}\text { Moser et al., } \\
2018\end{array}$ \\
\hline P. pastoris & Methanol** & squalene & $58 \mathrm{mg} / \mathrm{L}$ & \\
\hline P. pastoris & Methanol** & lycopene & $714 \mathrm{mg} / \mathrm{L}$ & $\begin{array}{l}\text { Zhang et al., } \\
2020\end{array}$ \\
\hline M. alcaliphilum & Methane & $\alpha$-humulene & $\begin{array}{l}0.75 \mathrm{mg} / \mathrm{g} \\
\text { CDW }\end{array}$ & $\begin{array}{l}\text { Nguyen et al., } \\
2020\end{array}$ \\
\hline M. acetivorans & Methanol* & isoprene & $0.954 \mathrm{mM} / \mathrm{L}$ & $\begin{array}{l}\text { Aldridge et al., } \\
2021\end{array}$ \\
\hline M. barkeri & Methanol* & isoprene & $36.0 \mu \mathrm{M} / \mathrm{L}$ & \\
\hline
\end{tabular}

*Complex medium; **Carbon sources switched from glycerol to methanol in fed-batch fermentation.

de novo production using heterologously expressed $\mathrm{CBB}$ cycle enzymes, overexpressed native genes in the XuMP cycle, and deletions of certain fatty acid enzymes. The mutant was ultimately capable of autotrophic growth on $\mathrm{CO}_{2}$ (Gassler et al., 2020). Likewise, artificial methanotrophy and formatotrophy have been explored in a complex rewiring of E. coli (Bennett et al., 2018; Chen et al., 2020; Kim et al., 2020). These seminal works are excellent examples of how systems biology can be applied to tune precursors, adapt strains, and incorporate well-defined isoprenoid pathways for higher production efficiency at lower substrate costs.

\section{Phototrophic Isoprenoid Production}

Phototrophic growth is characterized by the photosynthetic conversion of $\mathrm{CO}_{2}$ to sugars via complex photoreductive reactions and the Calvin-Benson-Bassham cycle. Photosynthetic organisms naturally produce carotenoids in high concentrations to cope with excess intracellular reactive oxygen species. Specifically, lutein is used in non-photochemical quenching of chlorophyll triplets during photosynthesis (Dall'Osto et al., 2006), zeaxanthin for heat dissipation and photoprotection, while carotene and chlorophyll absorb light. In many cases, gains from the engineering of the genetic architecture of photosynthesis (e.g., light harvesting complexes, RuBisCo) have been limited. Nonetheless, cyanobacteria, which only exhibit the MEP pathway, have become major targets for metabolic engineering due to their genetic plasticity and malleability with respect to isoprenoid precursors, especially through carbon sinks.

Engineered carbon sinks operate on the hypothesis that carbon fixation reactions are faster than downstream carbon utilizing growth reactions such that the accumulation of intracellular carbon metabolites reduces NADPH consumption and ultimately inhibits photosynthesis (Oliver and Atsumi, 2015). Introduction of exogenous genes demonstrated a 1.8-fold increase in carbon yield for the generation of 2,3-butanediol
(Oliver and Atsumi, 2015). Others have shown sucrose, ethylene, and isobutyraldehyde production all while enhancing photosynthetic activity through more optimal use of the electron transfer chain (Ducat et al., 2012; Santos-Merino et al., 2021). This effect was also found to be additive when multiple sinks were introduced, suggesting that "sink engineering" could be conceptually applied to secondary metabolite synthesis through downstream modifications of the MEP pathway (SantosMerino et al., 2021). Several works have capitalized on this upregulation of photosynthesis by combining this source/sink approach with computationally informed modification of limonene synthase, resulting in a 100-fold production improvement in limonene production (Wang et al., 2016) and potential applications for other isoprenoids.

Cyanobacterial studies have also made improvements through direct modification of native isoprenoid pathway genes in combination with a product-specific terminal synthase. Synechococcus elongatus and Synechocystis sp. PCC 6803 have been primary targets for production with recent attempts focusing on generation of isoprene, with a hallmark study demonstrating $1.26 \mathrm{~g} / \mathrm{L}$ production (Gao et al., 2016; Yang et al., 2016) albeit over several weeks. This feat was accomplished through the overexpression of MEP pathway enzymes though, more importantly, by bioprospecting for a more efficient isoprene synthase. Comprehensive analyses of the MEP pathway metabolic bottlenecks in S. elongatus have also been studied by a systematic investigation of each enzymatic step in the MEP pathway, specifically using isoprene as a simple reporter for MEP flux (Englund et al., 2018). The work found that the regulatory circuitry of the S. elongatus MEP pathway is, like that of many other MEP pathway harboring organisms, complex and that a simple overexpression of select pathway genes does not necessarily equate to higher/lower production. Despite this complexity, products including squalene (Pattanaik et al., 2020), bisabolene (Sebesta and Peebles, 2020; Rodrigues and Lindberg, 2021), and $\alpha$-farnesene (Lee et al., 2017) have been produced in $S$. elongatus through some combination of $i d i, d x s$, isp $A$, and terminal synthase overexpression. It is possible that cyanobacteria could benefit from acetyl-CoA/pyruvate precursor rebalancing. In one study, overexpression of a pyruvate dehydrogenase increased the pool of available acetyl-CoA for isopropanol production (Hirokawa et al., 2020) and could, in theory, be applied to facilitate a heterologously expressed MVA pathway or, in the reverse, to enhance pyruvate accumulation.

Unlike cyanobacteria, eukaryotic algae maintain both the MVA pathway, located in the cytosol, and the MEP pathway, which is sequestered to the chloroplast in proximity to $\mathrm{CO}_{2}$ derived metabolites from photosynthesis. Algae have been hailed as candidate bioproduction microbes for many years due to their propagation in many media and thus potential for growth in wastewater streams like agricultural runoff rich in phosphorus and nitrogen. In general, however, eukaryotic algae are notably more challenging to engineer due to their comparably smaller metabolic toolkits and robust regulatory mechanisms on metabolic flux. Studies have established their propensity for some algal isoprenoid production in low titers including patchoulol (Lauersen et al., 2016), bisabolene (Wichmann et al., 
2018), and mixed diterpenoids (Lauersen et al., 2018) in the modal alga Chlamydomonas reinhardtii with $\mathrm{CO}_{2}$ as the sole carbon source. Another alga, Dunaliella salina, has been singled out due to its resilience to highly saline environments that serve as a natural antibiotic against contaminants like protozoa, bacteria, dinoflagellates, and other algae. D. salina also naturally accumulates $\beta$-carotene under abiotic stress and remains one of the few commercially exploited green algae (Borowitzka, 2013; Fachet et al., 2020) along with Haematococcus pluvialis for astaxanthin production. Lastly, Botryococcus braunii, a colonial green alga, is rich in isoprenoid derived lipids that consist of $35 \%$ dry cell weight (DCW) biomass. The isoprenoids generated are characterized by race and consist of either Botryococcenes (C30-C37), methylated squalenes (C31-C34), or odd-number n-alkadienes or trienes (C23-C33; Metzger and Largeau, 2005). Despite their unique composition, broad attempts to culture and optimize isoprenoid production have been limited in part due to slow growth comparative to other green algae (Morales-Sánchez et al., 2017). Somewhat remarkably, both commercial successes stem from unmodified organisms that simply generate isoprenoids under abiotic stress conditions.

Diatoms are a unique subset of algae with a characteristic cell-wall composed of silica. Certain diatoms are capable of generating highly branched isoprenoids (HBIs) like trienes, tetraenes, and pentaenes intrinsic to some diatoms with potential for pharmaceutical or biofuel usage (Athanasakoglou et al., 2019), possibly generated by promiscuous activity of diatom specific farnesyl pyrophosphate synthases (Ferriols et al., 2015). A specific diatom, Haslea ostrearia maintains a plastidal MEP cycle with a cytosolic MVA pathway and has demonstrated significant crosstalk between these localized elements, suggesting complex regulatory mechanisms perhaps in response to external stimuli and pose potential opportunities to tune both pathways for downstream C5 precursors depending on target terpenoids.

A final distinctive group of phototrophic organisms are purple non-sulfur bacteria, which are identified by a unique color that stems from a combination of pigmented carotenoids. In particular, Rhodobacter sphaeroides is a well-established isoprenoid producer, with industrial production of sesquiterpenes valencene and nootkanone demonstrated by BASF (Beekwilder et al., 2014; Schempp et al., 2018). Like many bacteria, $R$. sphaeroides accumulates polyhydroxybutyrate (PHB), a biopolymer with industrial bioplastic applications in of itself, under nitrogen limited conditions. Elimination of the $\mathrm{PHB}$ biosynthetic pathway (phaC1, phaC2) and expression of the heterologous MVA pathway contributed to increased flux through the isoprenoid pathway under nitrogen limited conditions (Orsi et al., 2020b).

As a whole, photosynthetic organisms remain tantalizingly elusive for high titer heterologous isoprenoid production despite advances in "sink engineering" and successes in the production of certain short chain biofuels.

\section{Formatotrophic Production Pathways}

Formate remains an enticing $\mathrm{C} 1$ substrate due to the relative ease with which it may be generated. Proposed strategies include the hydration of syngas, the hydrogenation of $\mathrm{CO}_{2}$, and electrochemical reduction of $\mathrm{CO}_{2}$ using, preferably, renewable generated electricity (Yishai et al., 2016). Bioproduction on formate remains challenging, though recent works have attempted to address this challenge by mapping natural pathways within the context of microbial metabolism (Bar-Even, 2016). The intrinsic nature of formate as an intermediate and availability of natural formate assimilation pathways like the serine, reductive acetyl-CoA, RuMP, XuMP, and reductive glycine pathways have led to the proposal of many synthetic pathways that could theoretically outperform their natural counterparts (Bar-Even, 2016). This hypothesis was encouraged by a previous study that determined formate, not formaldehyde, was the major branch point in $M$. extorquens methylotrophy (Crowther et al., 2008). In particular, this suggested that direct feeding of formate could be energetically beneficial due to the affiliated reduction of $\mathrm{NAD}^{+}$in aldehyde dehydrogenase thereby further supporting formatotrophic pathways (Crowther et al., 2008).

Acting on this hypothesis, $M$. extorquens genes encoding formate-THF ligase, methenyl-THF cyclohydrolase, and methylene-THF dehydrogenase were heterologously expressed in $E$. coli to enable growth on formate through the serine cycle. In combination with downstream modifications, the strain was capable of $90 \mathrm{mg} / \mathrm{L}$ ethanol production on sugar-free formate minimal medium by adaptive laboratory evolution (ALE; Kim et al., 2019). In a subsequent study, expression of the reductive glycine pathway (rGlyP) in E. coli enabled growth on methanol and formate (Kim et al., 2020). Despite clear demonstration of formate-based growth here and $M$. extorquens isoprenoid production on methanol above, few formate derived isoprenoid compounds have been shown. A single exception was a study of the archaea Methanococcus maripaludis, which is capable of growth on $\mathrm{H}_{2}, \mathrm{CO}_{2}$, formate, and acetate as substrates under strict anaerobic conditions. Heterologous expression of a geraniol synthase enabled production of $4.0 \mathrm{mg} / \mathrm{g}$ and $2.8 \mathrm{mg} / \mathrm{g}$ geraniol on $\mathrm{H}_{2} / \mathrm{CO}_{2}$ and formate feeds, respectively (Lyu et al., 2016). Although meager, this represents a baseline for further isoprenoid production and, with the addition of the groundbreaking production of formatotrophic E. coli works, likely represents the first of many formate-based production strains.

So far, we have described a number of routes for isoprenoid production on C1 substrates, including several instances in which whole pathways have been translated between organisms. Life cycle assessment (LCA) and technoeconomic analysis (TEA) will both be critical in quantifying the relative process level sustainability and monetary impacts, validating whether modified microbes are competitive with conventional production on glucose or from petroleum, and prioritizing future optimization opportunities based on projected impact gains. Growth and production on $\mathrm{C} 1$ substrates are inherently more sustainable than on pure sugar substrates, however the sustainability of the entire process from cradle-to-gate will be contingent on nontrivial improvement of production titer, rate, and yield. While LCAs and TEAs are common in $\mathrm{CO}_{2}$-derived biofuel production, they remain uncommon for all other $\mathrm{C} 1$ substrates. Indeed, the first LCA/TEA of a methane-derived bioproduct was only recently published (Fei et al., 2020). Nonetheless this 
initial study provides a baseline for future valorization of other C1-derived chemicals and, hopefully, represents a first effort to quantify the economic and sustainability advantages of C1 substrates.

\section{ISOPRENOID PRODUCTION ON LIGNOCELLULOSIC CARBON SOURCES}

Certain microbes are capable of valorizing more complex waste streams due to unique evolutionary predispositions. Here, we describe two strains of oleaginous yeasts, Yarrowia lipolytica and Rhodosporidium toruloides, capable of high titer isoprenoid production from woody biomass and waste cooking oil (WCO). Lastly, we describe two prototypical isoprenoid production platforms: $P$. putida, which is a prime candidate for conversion of pretreated lignocellulosic biomass, and B. subtilis, a candidate bacteria renown for high titer protein production.

\section{Yarrowia lipolytica}

The oleaginous yeast Yarrowia lipolytica can naturally assimilate many atypical carbon sources including glycerol, organic acids, succinate, citrate, and even WCO. Likewise, Y. lipolytica is of keen interest due to its natural accumulation of $\beta$-carotene, farnesene, and linalool. Multi-copy pathway integration has proven especially successful in targeted isoprenoid overproduction (Xie et al., 2015). A recent work applied a random chromosomal integration approach of multiple MVA pathway operons, cofactor modulation, and culture condition tuning produced $25.55 \mathrm{~g} / \mathrm{L}$ $\alpha$-farnesene on YPD complex medium over 20 days in fed-batch production with significant byproduct formation (Liu et al., 2019). A similar strategy led to $6.5 \mathrm{~g} / \mathrm{L}$ production of $\beta$-carotene by chromosomal integration of multiple copies of CarB, CarRP, and GGPPS in fed-batch production with over $40 \mathrm{~g} / \mathrm{L}$ lipid byproduct (Larroude et al., 2018). Other reports of note include high squalene production at titers of $531.6 \mathrm{mg} / \mathrm{L}$ (Gao et al., 2017) and $402.4 \mathrm{mg} / \mathrm{L}$ (Arnesen et al., 2020). Building upon previous limonene demonstrations with neryl diphosphate synthase (tNPPS1) from Agastache rugosa (Korean mint) and limonene synthase from Solanum lycopersicum (tomato; Cao et al., 2016), Y. lipolytica ultimately yielded $165.3 \mathrm{mg} / \mathrm{L}$ limonene on glycerol/citrate (Cheng et al., 2019). More comprehensive descriptions of $Y$. lipolytica regulatory changes for production have also been published (Arnesen et al., 2020).

$Y$. lipolytica is also capable of converting fatty acids into C2 substrates through the beta-oxidation pathway and has high native lipid tolerance. Recent works have demonstrated high lipid production of modified Y. lipolytica on pretreated lignocellulosic biomass ( $0.11 \mathrm{~g}$ lipids/g sugars), even approaching efficiencies observed on glucose (Yook et al., 2020). In fact, Y. lipolytica has shown up to $90 \%$ DCW lipid accumulation (Park et al., 2018), which demonstrates an encouraging propensity for lipid tolerance. This tolerance has been harnessed by works that have grown Y. lipolytica strains on WCO. Growth on WCO increased lipolytic activity (Domínguez et al., 2010) and, in one study, a Y. lipolytica strain expressed D-limonene synthase
(Citrus limon) and L-limonene synthase (Mentha spicata) to yield $2.4 \mathrm{mg} / \mathrm{L}$ of each enantiomer on WCO (Pang et al., 2019). Although this strain has produced only $11 \mathrm{mg} / \mathrm{L}$ of each enantiomer on complex medium, this stands as an excellent proof of concept for future ALE and optimization studies on WCO.

Xylose catabolism and overcoming catabolite repression are major boundaries to bioproduction on lignocellulosic biomass (Sun et al., 2021). One study showed that carbon source switching enabled production of $20.6 \mathrm{mg} / \mathrm{L}$ and $15.1 \mathrm{mg} / \mathrm{L}$ limonene in Y. lipolytica from xylose and a $50 \%$ lignocellulosic biomass 50\% YP rich medium broth, respectively (Yao et al., 2020). This feat was accomplished by overexpression of a native xylulose synthase with heterologous expression of xylitol dehydrogenase and xylulose reductase from Scheffersomyces stipitis (Yao et al., 2020). Together, these modifications provided increased G3P production and, ultimately, increased flux through the MVA pathway.

\section{Rhodosporidium toruloides}

$R$. toruloides has attracted attention due to natural high titer lipid and carotenoid accumulation, namely torularhodin, torulene, $\gamma$-carotene, and $\beta$-carotene, as a convenient carbon storage mechanism under nitrogen-limited conditions (Park et al., 2018). Originally isolated from wood pulp, $R$. toruloides can also metabolize many components of lignocellulosic biomass and has shown simultaneous uptake not only of pentose and hexose sugars, but of p-coumaric acid and aromatic motifs analogous to lignin, which suggest that it could be adapted for direct consumption of lignin (Yaegashi et al., 2017). These traits are further complemented by its ability to thrive on various pretreatment conditions. For example, growth has been demonstrated on ionic liquid (choline $\alpha$-ketoglutarate) and alkaline pretreated cellulosic biomass, with the latter accumulating $680 \mathrm{mg} / \mathrm{L} \alpha$-bisabolene in fed-batch reactor conditions (Yaegashi et al., 2017). Further optimization of the $\alpha$-bisabolene synthase cassette yielded 4-fold increased titer on lignocellulosic biomass, reaching a final titer of $2.2 \mathrm{~g} / \mathrm{L}$ on corn stover hydrolysate (Kirby et al., 2021). 1,8-cineole was also accumulated to a titer of $1.4 \mathrm{~g} / \mathrm{L}$ on the same substrate, both of which represent titers that, even without significant core metabolic rewiring or downregulation, outstrip comparative E. coli and S. cerevisiae production. Importantly, pilot scaling of $R$. toruloides to a $1,000 \mathrm{~L}$ bioreactor for lipid production has been successfully shown (Soccol et al., 2017). Collectively, these traits establish $R$. toruloides as a potential microbial host for lignin valorization. The translation of successful pilot scale $R$. toruloides lipid production platforms to strains with tuned lipid reflex pathways could elevate the yeast to an industrially competitive isoprenoid production platform.

\section{Pseudomonas putida}

As noted with $Y$. lipolytica, tolerance to and simultaneous uptake of multiple carbon substrates is a key phenotype for successful bioproduction on lignocellulosic biomass. The soil bacterium Pseudomonas putida maintains significant advantages 
over common production chassis due to its natural biodegradation pathways and oxidative stress tolerance, which has contributed to its broad proliferation in many environmental niches. Several studies have explored substrate tolerance through toxicity adaptive laboratory evolution (TALE) of $P$. putida (Mohamed et al., 2020; Lim et al., 2021). A recent work integrated three different xylose pathways (Dahms, Isomerase, and Weimberg) on plasmids to enable growth on xylose, a prominent component of degraded hemicellulose (Bator et al., 2019). The combination of pathway expression and ALE resulted in improved tolerance and hence improved growth rate (Bator et al., 2019).

Other production schemes have exploited the natural aromatic tolerance of $P$. putida for growth on substrates like toluene, m-xylene, and p-xylene (Nikel and de Lorenzo, 2018). Comparatively, $P$. putida maintains better de novo tolerance toward products that are typically toxic to other organisms (Mi et al., 2014). For example, the saprophytic uptake of organic nutrients and high tolerance to oxidative stress is ideal for biofuel production candidates (Kim and Park, 2014). These traits coupled with overexpression of efflux pumps have shown increased tolerance to short chain C4 and C5 alcohols, which could prove especially valuable for production of isoprenoid biofuels (Basler et al., 2018).

In a hallmark bioproduction study, $2.21 \mathrm{~g} / \mathrm{L}$ of mevalonate were generated by $P$. putida in M9 minimal medium supplemented with $7.5 \mathrm{~g} / \mathrm{L}$ 2,3-butanediol by overexpression of the upper MVA pathway enzymes, namely the native AtoB and the MvaE/ MvaS from Enterococcus faecalis (Yang et al., 2020). Mevalonate production on 2,3-butanediol proved 6.61- and 8.44-fold higher than production on glucose and glycerol, respectively, though with manageable growth inhibition (Yang et al., 2020). Overall, P. putida isoprenoid production has historically been limited to zeaxanthin and geranic acid such that only recently have studies begun addressing MEP/heterologous MVA precursor limitations. One such study exhibited metabolic rerouting of central carbon metabolism from the EMP to ED cycles for better precursor management, namely efficient pyruvate production (Sánchez-Pascuala et al., 2019). This strategy led to a 2-fold increase in carotenoid yield on glucose with plasmid expression of a lycopene synthesis pathway but without any modification to the endogenous MEP pathway (Sánchez-Pascuala et al., 2019).

It is clear that $P$. putida has high innate tolerance to toxic substrates and, as in nature, can adapt to adverse growth conditions. The next, critical stages of realizing $P$. putida as a chemical production platform will be combining advances in ALE, precursor availability, and pathway tuning to enhance terpene synthesis on atypical carbon substrates.

\section{Bacillus subtilis}

Bacillus subtilis is one of the best characterized gram-positive bacteria to date and has been an attractive bioproduction candidate due to high titer protein production and high secreting properties. Largely, the industrial focus has been on the production of biologics and enzymes (Pham et al., 2019). B. subtilis maintains a faster growth rate than $S$. cerevisiae, a robust metabolism on diverse carbon substrates, and has also shown natural isoprene production at titers comparable to $E$. coli (Zhang et al., 2015). Unlike other chassis organisms like P. putida, B. subtilis is generally recognized as safe (GRAS), a designation that reduces regulatory boundaries to commercialization. Collectively, these factors suggest that $B$. subtilis could be an excellent candidate for isoprenoid production. Unfortunately, production studies remain relatively limited in part due to a poorly defined metabolic toolkit, which has historically been hampered by a limited subset of selection/ counterselection markers that have made genetic manipulation challenging.

Mirroring in E. coli from the early 2000s, recent production studies demonstrated that incorporation of amorphadiene synthase (ADS) with overexpression of DXS and IDI led to the accumulation of $20 \mathrm{mg} / \mathrm{L}$ amorphadiene (Zhou et al., 2013). This titer has dramatically improved to $116 \mathrm{mg} / \mathrm{L}$ using a CRISPR-cas9 system without culture medium optimization (Song et al., 2021) and then to $416 \mathrm{mg} / \mathrm{L}$ (Pramastya et al., 2021) with pyruvate supplementation. Another recent study overexpressed the entire MEP pathway excluding IDI, a taxadiene synthase, and a heterologous GGPPS in B. subtilis, leading to an accumulation of $17.8 \mathrm{mg} / \mathrm{L}$ taxadiene (Abdallah et al., 2019). Expression of a squalene synthase from Bacillus megaterium also enabled $7.5 \mathrm{mg} / \mathrm{L}$ production of squalene, which can serve as a precursor to other triterpenoids (Song et al., 2020). Although far from competitive with E. coli and S. cerevisiae, these initial demonstrations have provided a basis of isoprenoid production in B. subtilis. The publication by Song et al. is of particular interest due to their application of CRISPR-cas9 to circumvent boundaries that have historically limited the establishment of $B$. subtilis as an isoprenoid production workhorse. In theory, this approach could be easily translated to the production of other isoprenoid targets.

\section{PERSPECTIVES AND CONCLUSION}

The rapid expansion of -omics studies, deep sequencing, and pathway engineering have facilitated bioprospecting of more efficient enzymes, robust combinatorial approaches for tailored isoprenoid production, and the design of altogether novel production pathways. Such tools have also facilitated the exploration of plant derived CYPs and terminal synthases whose subsequent expression has expanded the microbial isoprenoid repertoire to more pharmacologically relevant as well as entirely synthetic terpenoids. In this review, we focused on improvements to isoprenoid precursor biosynthesis and translation of enzymes or pathways between organisms, which could assist in overcoming current major barriers to commercial viability (Zu et al., 2020). Specifically, we highlighted how atypical carbon sources and non-model organisms harbor metabolic advantages that could be harnessed to reduce substrate costs and the associated emissions of bioproduction. Co-substrate utilization by certain organisms as in the case of $R$. toruloides and $P$. putida has the potential to unlock lignocellulosic biomass and many 
methylotrophs could tap into inexpensive and highly abundant substrates.

We have also described works that capitalized upon the modularity of isoprenoid advances through heterologous expression of entire pathways. Systems engineering strategies are of particular interest for $\mathrm{C} 1$ metabolism. The translation of successful whole systems engineering strategies from $E$. coli and S. cerevisiae to non-model organisms will prove useful in further optimization. For example, the entire MVA pathway had been expressed in E. coli many years ago (Martin et al., 2003) and a decade later, the entire MEP pathway has been expressed in S. cerevisiae conversely (Kirby et al., 2016). Both strains have also been extensively mapped through metabolic flux analysis (MFA) which has proven pivotal in metabolic engineering (Orth et al., 2010). The translation of systems engineering strategies like MFA and genome-scale modeling to other organisms will undoubtedly help to inform and improve isoprenoid production in non-model organisms. An MFA of $R$. sphaeroides, for example, showed a mutualistic coupling between its MEP and MVA pathways (Orsi et al., 2020a). Remarkably the true extent of the MEP pathway MVA pathway relationship could not be resolved as gene knockouts tended to have unpredictable effects on $\mathrm{C} 13$ product partitioning but suggested complex regulatory interactions. Nonetheless further work could shed light on how such combined MVA/MEP pathway systems could prove beneficial (Orsi et al., 2020a). Similarly, a metabolic flux reconstruction of Dunaliella salina established baseline carbon metabolism during carotenogenesis (Fachet et al., 2020), a critical step in elucidating metabolic bottlenecks. TALE has also proven a powerful strategy for increasing resistance to toxicity of high titer products especially with alcohols. TALE has now been applied to P. putida and enhanced toxicity tolerance against the lignocellulosic aromatics such as p-coumaric acid and ferulic acid (Lim et al., 2020; Mohamed et al., 2020). The application of machine learning approaches has enabled extrapolation and gap filling in genome-scale models for rationally designed engineering strategies of non-canonical organisms, as demonstrated to great effect in Y. lipolytica (Czajka et al., 2021). And, finally, C1 assimilation pathways have been thoroughly explored, synthetic and natural routes hypothesized (Bar-Even, 2016), then optimal pathways have been heterologously expressed in conventional production chassis (Kim et al., 2020). Having shown adapted growth on C1 substrates there is now a tremendous opportunity to further develop strains for isoprenoid production especially given the comparative sustainability and cost reduction of such substrates with respect to production on refined sugars.

Consortial approaches are also valuable by improving total system productivity. Microbial consortia have proven successful for short chain alcohol production from lignocellulosic biomass (Minty et al., 2013) and have recently been explored in the cross-feeding of methane-derived organic acids produced by Methylococcus capsulatus to E. coli for the generation of mevalonate at $60 \mathrm{mg} / \mathrm{L}$ (Lee et al., 2021). Building upon CYP optimization, an E. coli and S. cerevisiae consortium produced $33 \mathrm{mg} / \mathrm{L}$ oxygenated taxanes in a consortia where E. coli consumes xylose and produces acetate and the precursor taxadiene for consumption and further conjugation in S. cerevisiae, respectively (Zhou et al., 2015). Another group produced $0.32 \mathrm{~g}$ mevalonate $/ \mathrm{g}$ ethanol in $P$. putida batch experiments (Yang et al., 2019) that, paired with the aforementioned successes in ALE, could provide another promising cross-feeding consortial bioproduction strategy. Finally, isoprenoid production has been expanded to 2,3-butanediol (Yang et al., 2020), which could facilitate consortial bioproduction by subdividing pathways between members.

Exploration of the microbial tree of life has continued to yield an abundant natural diversity of protein homologues, pathway shunts, and mechanisms with which targeted production of isoprenoids has been demonstrably improved. The principal challenge of isoprenoid bioproduction in the next decade will be bridging the knowledge gap between conventional high titer bioproduction on pure sugar substrates and non-model comparatively low titer production on affordable substrates.

\section{AUTHOR CONTRIBUTIONS}

DC and TSL contributed to the writing of this review and conceived the topic. DC prepared the initial draft, reviewed, and edited the draft. TSL directed the writing process and wrote and reviewed the draft. All authors contributed to the article and approved the submitted version.

\section{FUNDING}

This work was supported by the DOE Joint BioEnergy Institute (http://www.jbei.org), supported by the US Department of Energy, Office of Science, Office of Biological and Environmental Research, through contract DE-AC0205CH11231 with Lawrence Berkeley National Laboratory.

\section{ACKNOWLEDGMENTS}

The United States Government retains and the publisher, by accepting the article for publication, acknowledges that the United States Government retains a non-exclusive, paid-up, irrevocable, worldwide license to publish or reproduce the published form of this manuscript, or allow others to do so, for United States Government purposes. The views and opinions of the authors expressed herein do not necessarily state or reflect those of the United States Government or any agency thereof. Neither the United States Government nor any agency thereof, nor any of their employees, makes any warranty, expressed or implied, or assumes any legal liability or responsibility for the accuracy, completeness, or usefulness of any information, apparatus, product, or process disclosed, or represents that its use would not infringe privately owned rights. 


\section{REFERENCES}

Abdallah, I. I., Pramastya, H., van Merkerk, R., and Sukrasno, and Quax, W. J., (2019). Metabolic engineering of Bacillus subtilis toward taxadiene biosynthesis as the first committed step for taxol production. Front. Microbiol. 10:218. doi: $10.3389 /$ fmicb.2019.00218

Aldridge, J., Carr, S., Weber, K. A., and Buan, N. R. (2021). Anaerobic production of isoprene by engineered methanosarcina species archaea. Appl. Environ. Microbiol. 87, e02417-e02420. doi: 10.1128/AEM.02417-20

Alonso-Gutierrez, J., Chan, R., Batth, T. S., Adams, P. D., Keasling, J. D., Petzold, C. J., et al. (2013). Metabolic engineering of Escherichia coli for limonene and perillyl alcohol production. Metab. Eng. 19, 33-41. doi: 10.1016/j. ymben.2013.05.004

Arnesen, J. A., Kildegaard, K. R., Cernuda Pastor, M., Jayachandran, S., Kristensen, M., and Borodina, I. (2020). Yarrowia lipolytica strains engineered for the production of terpenoids. Front. Bioeng. Biotechnol. 8:945. doi: 10.3389/ fbioe.2020.00945

Athanasakoglou, A., Grypioti, E., Michailidou, S., Ignea, C., Makris, A. M., Kalantidis, K., et al. (2019). Isoprenoid biosynthesis in the diatom Haslea ostrearia. New Phytol. 222, 230-243. doi: 10.1111/nph.15586

Bar-Even, A. (2016). Formate assimilation: the metabolic architecture of natural and synthetic pathways. Biochemistry 55, 3851-3863. doi: 10.1021/acs. biochem.6b00495

Basler, G., Thompson, M., Tullman-Ercek, D., and Keasling, J. (2018). A Pseudomonas putida efflux pump acts on short-chain alcohols. Biotechnol. Biofuels 11:136. doi: 10.1186/s13068-018-1133-9

Bator, I., Wittgens, A., Rosenau, F., Tiso, T., and Blank, L. M. (2019). Comparison of three xylose pathways in Pseudomonas putida KT2440 for the synthesis of valuable products. Front. Bioeng. Biotechnol. 7:480. doi: 10.3389/ fbioe.2019.00480

Beekwilder, J., van Houwelingen, A., Cankar, K., van Dijk, A. D. J., de Jong, R. M., Stoopen, G., et al. (2014). Valencene synthase from the heartwood of Nootka cypress (Callitropsis nootkatensis) for biotechnological production of valencene. Plant Biotechnol. J. 12, 174-182. doi: 10.1111/pbi.12124

Bennett, R. K., Gonzalez, J. E., Whitaker, W. B., Antoniewicz, M. R., and Papoutsakis, E. T. (2018). Expression of heterologous non-oxidative pentose phosphate pathway from Bacillus methanolicus and phosphoglucose isomerase deletion improves methanol assimilation and metabolite production by a synthetic Escherichia coli methylotroph. Metab. Eng. 45, 75-85. doi: 10.1016/j. ymben.2017.11.016

Biggs, B. W., Lim, C. G., Sagliani, K., Shankar, S., Stephanopoulos, G., De Mey, M., et al. (2016). Overcoming heterologous protein interdependency to optimize P450-mediated Taxol precursor synthesis in Escherichia coli. Proc. Natl. Acad. Sci. U. S. A. 113, 3209-3214. doi: 10.1073/pnas.1515826113

Bitok, J. K., and Meyers, C. F. (2012). 2C-methyl-d-erythritol 4-phosphate enhances and sustains cyclodiphosphate synthase IspF activity. ACS Chem. Biol. 7, 1702-1710. doi: 10.1021/cb300243w

Borowitzka, M. A. (2013). High-value products from microalgae-their development and commercialisation. J. Appl. Phycol. 25, 743-756. doi: 10.1007/ s10811-013-9983-9

Boucher, Y., Kamekura, M., and Doolittle, W. F. (2004). Origins and evolution of isoprenoid lipid biosynthesis in archaea. Mol. Microbiol. 52, 515-527. doi: $10.1111 / \mathrm{j} .1365-2958.2004 .03992 . x$

Caforio, A., and Driessen, A. J. M. (2017). Archaeal phospholipids: structural properties and biosynthesis. Biochim. Biophys. Acta Mol. Cell Biol. Lipids 1862, 1325-1339. doi: 10.1016/j.bbalip.2016.12.006

Cao, X., Lv, Y.-B., Chen, J., Imanaka, T., Wei, L.-J., and Hua, Q. (2016). Metabolic engineering of oleaginous yeast Yarrowia lipolytica for limonene overproduction. Biotechnol. Biofuels 9:214. doi: 10.1186/s13068-016-0626-7

Castelle, C. J., and Banfield, J. F. (2018). Major new microbial groups expand diversity and alter our understanding of the tree of life. Cell 172, 1181-1197. doi: $10.1016 /$ j.cell.2018.02.016

Chang, M. C. Y., Eachus, R. A., Trieu, W., Ro, D.-K., and Keasling, J. D. (2007). Engineering Escherichia coli for production of functionalized terpenoids using plant P450s. Nat. Chem. Biol. 3, 274-277. doi: 10.1038/nchembio875

Chappell, J. (1995). The biochemistry and molecular biology of isoprenoid metabolism. Plant Physiol. 107, 1-6. doi: 10.1104/pp.107.1.1
Chatzivasileiou, A. O., Ward, V., Edgar, S. M., and Stephanopoulos, G. (2019). Two-step pathway for isoprenoid synthesis. Proc. Natl. Acad. Sci. U. S. A. 116, 506-511. doi: 10.1073/pnas.1812935116

Chen, F. Y.-H., Jung, H.-W., Tsuei, C.-Y., and Liao, J. C. (2020). Converting Escherichia coli to a synthetic methylotroph growing solely on methanol. Cell 182, 933-946.e14. doi: 10.1016/j.cell.2020.07.010

Cheng, B.-Q., Wei, L.-J., Lv, Y.-B., Chen, J., and Hua, Q. (2019). Elevating limonene production in oleaginous yeast Yarrowia lipolytica via genetic engineering of limonene biosynthesis pathway and optimization of medium composition. Biotechnol. Bioprocess Eng. 24, 500-506. doi: 10.1007/ s12257-018-0497-9

Clomburg, J. M., Crumbley, A. M., and Gonzalez, R. (2017). Industrial biomanufacturing: the future of chemical production. Science 355:aag0804. doi: 10.1126/science.aag0804

Clomburg, J. M., Qian, S., Tan, Z., Cheong, S., and Gonzalez, R. (2019). The isoprenoid alcohol pathway, a synthetic route for isoprenoid biosynthesis. Proc. Natl. Acad. Sci. U. S. A. 116, 12810-12815. doi: 10.1073/pnas.1821004116

Conrado, R. J., and Gonzalez, R. (2014). Chemistry. Envisioning the bioconversion of methane to liquid fuels. Science 343, 621-623. doi: 10.1126/science.1246929

Crowther, G. J., Kosály, G., and Lidstrom, M. E. (2008). Formate as the main branch point for methylotrophic metabolism in Methylobacterium extorquens AM1. J. Bacteriol. 190, 5057-5062. doi: 10.1128/JB.00228-08

Czajka, J. J., Oyetunde, T., and Tang, Y. J. (2021). Integrated knowledge mining, genome-scale modeling, and machine learning for predicting Yarrowia lipolytica bioproduction. Metab. Eng. 67, 227-236. doi: 10.1016/j. ymben.2021.07.003

D’Adamo, S., Schiano di Visconte, G., Lowe, G., Szaub-Newton, J., Beacham, T., Landels, A., et al. (2019). Engineering the unicellular alga Phaeodactylum tricornutum for high-value plant triterpenoid production. Plant Biotechnol. J. 17, 75-87. doi: $10.1111 / \mathrm{pbi} .12948$

Dall'Osto, L., Lico, C., Alric, J., Giuliano, G., Havaux, M., and Bassi, R. (2006). Lutein is needed for efficient chlorophyll triplet quenching in the major LHCII antenna complex of higher plants and effective photoprotection in vivo under strong light. BMC Plant Biol. 6:32. doi: 10.1186/1471-2229-6-32

Della Monica, F., and Kleij, A. W. (2020). From terpenes to sustainable and functional polymers. Polym. Chem. 11, 5109-5127. doi: 10.1039/D0PY00817F

Dellas, N., Thomas, S. T., Manning, G., and Noel, J. P. (2013). Discovery of a metabolic alternative to the classical mevalonate pathway. elife 2:e00672. doi: $10.7554 /$ eLife.00672

Domínguez, A., Deive, F. J., Angeles Sanromán, M., and Longo, M. A. (2010). Biodegradation and utilization of waste cooking oil by Yarrowia lipolytica CECT 1240. Eur. J. Lipid Sci. Technol. 112, 1200-1208. doi: 10.1002/ejlt.201000049

Drummond, L., Kschowak, M. J., Breitenbach, J., Wolff, H., Shi, Y.-M., Schrader, J., et al. (2019). Expanding the isoprenoid building block repertoire with an IPP methyltransferase from Streptomyces monomycini. ACS Synth. Biol. 8, 1303-1313. doi: 10.1021/acssynbio.8b00525

Ducat, D. C., Avelar-Rivas, J. A., Way, J. C., and Silver, P. A. (2012). Rerouting carbon flux to enhance photosynthetic productivity. Appl. Environ. Microbiol. 78, 2660-2668. doi: 10.1128/AEM.07901-11

Dugar, D., and Stephanopoulos, G. (2011). Relative potential of biosynthetic pathways for biofuels and bio-based products. Nat. Biotechnol. 29, 1074-1078. doi: $10.1038 /$ nbt.2055

Dunlop, M. J. (2011). Engineering microbes for tolerance to next-generation biofuels. Biotechnol. Biofuels 4:32. doi: 10.1186/1754-6834-4-32

Dunlop, M. J., Dossani, Z. Y., Szmidt, H. L., Chu, H. C., Lee, T. S., Keasling, J. D., et al. (2011). Engineering microbial biofuel tolerance and export using efflux pumps. Mol. Syst. Biol. 7:487. doi: 10.1038/msb.2011.21

Eiben, C. B., de Rond, T., Bloszies, C., Gin, J., Chiniquy, J., Baidoo, E. E. K. et al. (2019). Mevalonate pathway promiscuity enables noncanonical terpene production. ACS Synth. Biol. 8, 2238-2247. doi: 10.1021/acssynbio.9b00230

Eiben, C. B., Tian, T., Thompson, M. G., Mendez-Perez, D., Kaplan, N., Goyal, G., et al. (2020). Adenosine triphosphate and carbon efficient route to second generation biofuel isopentanol. ACS Synth. Biol. 9, 468-474. doi: 10.1021/ acssynbio.9b00402

Englund, E., Shabestary, K., Hudson, E. P., and Lindberg, P. (2018). Systematic overexpression study to find target enzymes enhancing production of terpenes in synechocystis PCC 6803, using isoprene as a model compound. Metab. Eng. 49, 164-177. doi: 10.1016/j.ymben.2018.07.004 
Fachet, M., Witte, C., Flassig, R. J., Rihko-Struckmann, L. K., McKie-Krisberg, Z., Polle, J. E. W., et al. (2020). Reconstruction and analysis of a carbon-core metabolic network for Dunaliella salina. BMC Bioinform. 21:1. doi: 10.1186/ s12859-019-3325-0

Fei, Q., Liang, B., Tao, L., Tan, E. C. D., Gonzalez, R., Henard, C., et al. (2020). Biological valorization of natural gas for the production of lactic acid: techno-economic analysis and life cycle assessment. Biochem. Eng. J. 158:107500. doi: 10.1016/j.bej.2020.107500

Ferriols, V. M. E. N., Yaginuma, R., Adachi, M., Takada, K., Matsunaga, S., and Okada, S. (2015). Cloning and characterization of farnesyl pyrophosphate synthase from the highly branched isoprenoid producing diatom Rhizosolenia setigera. Sci. Rep. 5:10246. doi: 10.1038/srep10246

Gao, X., Gao, F., Liu, D., Zhang, H., Nie, X., and Yang, C. (2016). Engineering the methylerythritol phosphate pathway in cyanobacteria for photosynthetic isoprene production from $\mathrm{CO}_{2}$. Energy Environ. Sci. 9, 1400-1411. doi: 10.1039/C5EE03102H

Gao, S., Tong, Y., Zhu, L., Ge, M., Zhang, Y., Chen, D., et al. (2017). Iterative integration of multiple-copy pathway genes in Yarrowia lipolytica for heterologous $\beta$-carotene production. Metab. Eng. 41, 192-201. doi: 10.1016/j. ymben.2017.04.004

Gassler, T., Sauer, M., Gasser, B., Egermeier, M., Troyer, C., Causon, T., et al. (2020). The industrial yeast Pichia pastoris is converted from a heterotroph into an autotroph capable of growth on CO2. Nat. Biotechnol. 38, 210-216. doi: 10.1038/s41587-019-0363-0

George, K. W., Thompson, M. G., Kang, A., Baidoo, E., Wang, G., Chan, L. J. G., et al. (2015). Metabolic engineering for the high-yield production of isoprenoid-based $C_{5}$ alcohols in E. coli. Sci. Rep. 5:11128. doi: 10.1038/srep11128

George, K. W., Thompson, M. G., Kim, J., Baidoo, E. E. K., Wang, G., Benites, V. T., et al. (2018). Integrated analysis of isopentenyl pyrophosphate (IPP) toxicity in isoprenoid-producing Escherichia coli. Metab. Eng. 47, 60-72. doi: 10.1016/j.ymben.2018.03.004

Guo, X., Sun, J., Li, D., and Lu, W. (2018). Heterologous biosynthesis of (+)-nootkatone in unconventional yeast' ' Yarrowia lipolytica. Biochem. Eng. J. 137, 125-131. doi: 10.1016/j.bej.2018.05.023

Guo, J., Zhou, Y. J., Hillwig, M. L., Shen, Y., Yang, L., Wang, Y., et al. (2013). CYP76AH1 catalyzes turnover of miltiradiene in tanshinones biosynthesis and enables heterologous production of ferruginol in yeasts. Proc. Natl. Acad. Sci. U. S. A. 110, 12108-12113. doi: 10.1073/pnas.1218061110

Hartner, F. S., and Glieder, A. (2006). Regulation of methanol utilisation pathway genes in yeasts. Microb. Cell Factories 5:39. doi: 10.1186/1475-2859-5-39

Hausjell, J., Halbwirth, H., and Spadiut, O. (2018). Recombinant production of eukaryotic cytochrome P450s in microbial cell factories. Biosci. Rep. 38:BSR20171290. doi: 10.1042/BSR20171290

Hayakawa, H., Motoyama, K., Sobue, F., Ito, T., Kawaide, H., Yoshimura, T., et al. (2018). Modified mevalonate pathway of the archaeon Aeropyrum pernix proceeds via trans-anhydromevalonate 5-phosphate. Proc. Natl. Acad. Sci. U. S. A. 115, 10034-10039. doi: 10.1073/pnas.1809154115

Helfrich, E. J. N., Lin, G.-M., Voigt, C. A., and Clardy, J. (2019). Bacterial terpene biosynthesis: challenges and opportunities for pathway engineering. Beilstein J. Org. Chem. 15, 2889-2906. doi: 10.3762/bjoc.15.283

Hernandez-Arranz, S., Perez-Gil, J., Marshall-Sabey, D., and Rodriguez-Concepcion, M. (2019). Engineering Pseudomonas putida for isoprenoid production by manipulating endogenous and shunt pathways supplying precursors. Microb. Cell Factories 18:152. doi: 10.1186/ s12934-019-1204-z

Hernandez-Ortega, A., Vinaixa, M., Zebec, Z., Takano, E., and Scrutton, N. S. (2018). A toolbox for diverse oxyfunctionalisation of monoterpenes. Sci. Rep. 8:14396. doi: 10.1038/s41598-018-32816-1

Hirokawa, Y., Kubo, T., Soma, Y., Saruta, F., and Hanai, T. (2020). Enhancement of acetyl-CoA flux for photosynthetic chemical production by pyruvate dehydrogenase complex overexpression in Synechococcus elongatus PCC 7942. Metab. Eng. 57, 23-30. doi: 10.1016/j.ymben.2019.07.012

Hu, B., and Lidstrom, M. E. (2014). Metabolic engineering of Methylobacterium extorquens AM1 for 1-butanol production. Biotechnol. Biofuels 7:156. doi: 10.1186/s13068-014-0156-0

Ignea, C., Pontini, M., Motawia, M. S., Maffei, M. E., Makris, A. M., and Kampranis, S. C. (2018). Synthesis of 11-carbon terpenoids in yeast using protein and metabolic engineering. Nat. Chem. Biol. 14, 1090-1098. doi: 10.1038/s41589-018-0166-5
Jain, S., Caforio, A., and Driessen, A. J. M. (2014). Biosynthesis of archaeal membrane ether lipids. Front. Microbiol. 5:641. doi: 10.3389/fmicb.2014.00641

Johnson, C. W., Salvachúa, D., Rorrer, N. A., Black, B. A., Vardon, D. R., St. John, P. C., et al. (2019). Innovative chemicals and materials from bacterial aromatic catabolic pathways. Joule 19, 1523-1537. doi: 10.1016/j. joule.2019.05.011

Kang, A., George, K. W., Wang, G., Baidoo, E., Keasling, J. D., and Lee, T. S. (2016). Isopentenyl diphosphate (IPP)-bypass mevalonate pathways for isopentenol production. Metab. Eng. 34, 25-35. doi: 10.1016/j. ymben.2015.12.002

Kang, A., Meadows, C. W., Canu, N., Keasling, J. D., and Lee, T. S. (2017). High-throughput enzyme screening platform for the IPP-bypass mevalonate pathway for isopentenol production. Metab. Eng. 41, 125-134. doi: 10.1016/j. ymben.2017.03.010

Kang, A., Mendez-Perez, D., Goh, E.-B., Baidoo, E. E. K., Benites, V. T., Beller, H. R., et al. (2019). Optimization of the IPP-bypass mevalonate pathway and fed-batch fermentation for the production of isoprenol in Escherichia coli. Metab. Eng. 56, 85-96. doi: 10.1016/j.ymben.2019.09.003

Karunanithi, P. S., and Zerbe, P. (2019). Terpene synthases as metabolic gatekeepers in the evolution of plant terpenoid chemical diversity. Front. Plant Sci. 10:1166. doi: 10.3389/fpls.2019.01166

Kim, S. K., Han, G. H., Seong, W., Kim, H., Kim, S.-W., Lee, D.-H., et al. (2016). CRISPR interference-guided balancing of a biosynthetic mevalonate pathway increases terpenoid production. Metab. Eng. 38, 228-240. doi: 10.1016/j.ymben.2016.08.006

Kim, S., Lindner, S. N., Aslan, S., Yishai, O., Wenk, S., Schann, K., et al. (2020). Growth of E. coli on formate and methanol via the reductive glycine pathway. Nat. Chem. Biol. 16, 538-545. doi: 10.1038/s41589-020-0473-5

Kim, J., and Park, W. (2014). Oxidative stress response in Pseudomonas putida. Appl. Microbiol. Biotechnol. 98, 6933-6946. doi: 10.1007/ s00253-014-5883-4

Kim, S.-J., Yoon, J., Im, D.-K., Kim, Y. H., and Oh, M.-K. (2019). Adaptively evolved Escherichia coli for improved ability of formate utilization as a carbon source in sugar-free conditions. Biotechnol. Biofuels 12:207. doi: 10.1186/s13068-019-1547-z

King, J. R., Woolston, B. M., and Stephanopoulos, G. (2017). Designing a new entry point into isoprenoid metabolism by exploiting fructose-6-phosphate aldolase side reactivity of Escherichia coli. ACS Synth. Biol. 6, 1416-1426. doi: $10.1021 /$ acssynbio.7b00072

Kirby, J., Dietzel, K. L., Wichmann, G., Chan, R., Antipov, E., Moss, N., et al. (2016). Engineering a functional 1-deoxy-D-xylulose 5-phosphate (DXP) pathway in Saccharomyces cerevisiae. Metab. Eng. 38, 494-503. doi: 10.1016/j. ymben.2016.10.017

Kirby, J., Geiselman, G. M., Yaegashi, J., Kim, J., Zhuang, X., Tran-Gyamfi, M. B., et al. (2021). Further engineering of $R$. toruloides for the production of terpenes from lignocellulosic biomass. Biotechnol. Biofuels 14:101. doi: 10.1186/ s13068-021-01950-w

Kirby, J., and Keasling, J. D. (2009). Biosynthesis of plant isoprenoids: perspectives for microbial engineering. Annu. Rev. Plant Biol. 60, 335-355. doi: 10.1146/ annurev.arplant.043008.091955

Kirby, J., Nishimoto, M., Chow, R. W. N., Baidoo, E. E. K., Wang, G., Martin, J., et al. (2015). Enhancing Terpene yield from sugars via novel routes to 1-deoxy-d-xylulose 5-phosphate. Appl. Environ. Microbiol. 81, 130-138. doi: 10.1128/AEM.02920-14

Kuhl, K. P., Cave, E. R., Abram, D. N., and Jaramillo, T. F. (2012). New insights into the electrochemical reduction of carbon dioxide on metallic copper surfaces. Energy Environ. Sci. 5:7050. doi: 10.1039/c2ee21234j

Larroude, M., Celinska, E., Back, A., Thomas, S., Nicaud, J.-M., and Ledesma-Amaro, R. (2018). A synthetic biology approach to transform Yarrowia lipolytica into a competitive biotechnological producer of $\beta$-carotene. Biotechnol. Bioeng. 115, 464-472. doi: 10.1002/bit.26473

Lauersen, K. J., Baier, T., Wichmann, J., Wördenweber, R., Mussgnug, J. H., Hübner, W., et al. (2016). Efficient phototrophic production of a high-value sesquiterpenoid from the eukaryotic microalga Chlamydomonas reinhardtii. Metab. Eng. 38, 331-343. doi: 10.1016/j.ymben.2016.07.013

Lauersen, K. J., Wichmann, J., Baier, T., Kampranis, S. C., Pateraki, I., Møller, B. L., et al. (2018). Phototrophic production of heterologous diterpenoids and a hydroxy-functionalized derivative from Chlamydomonas reinhardtii. Metab. Eng. 49, 116-127. doi: 10.1016/j.ymben.2018.07.005 
Lee, H., Baek, J. I., Lee, J.-Y., Jeong, J., Kim, H., Lee, D.-H., et al. (2021). Syntrophic co-culture of a methanotroph and heterotroph for the efficient conversion of methane to mevalonate. Metab. Eng. 67, 285-292. doi: 10.1016/j. ymben.2021.07.008

Lee, H. J., Lee, J., Lee, S.-M., Um, Y., Kim, Y., Sim, S. J., et al. (2017). Direct conversion of $\mathrm{CO}_{2}$ to $\alpha$-farnesene using metabolically engineered Synechococcus elongatus PCC 7942. J. Agric. Food Chem. 65, 10424-10428. doi: 10.1021/ acs.jafc.7b03625

Levisson, M., Araya-Cloutier, C., de Bruijn, W. J. C., van der Heide, M., Salvador López, J. M., Daran, J.-M., et al. (2019). Toward developing a yeast cell factory for the production of prenylated flavonoids. J. Agric. Food Chem. 67, 13478-13486. doi: 10.1021/acs.jafc.9b01367

Li, Q., Fan, F., Gao, X., Yang, C., Bi, C., Tang, J., et al. (2017). Balanced activation of IspG and IspH to eliminate MEP intermediate accumulation and improve isoprenoids production in Escherichia coli. Metab. Eng. 44, 13-21. doi: 10.1016/j.ymben.2017.08.005

Li, Z., Jiang, Y., Guengerich, F. P., Ma, L., Li, S., and Zhang, W. (2020). Engineering cytochrome P450 enzyme systems for biomedical and biotechnological applications. J. Biol. Chem. 295, 833-849. doi: 10.1016/ S0021-9258(17)49939-X

Liang, W.-F., Cui, L.-Y., Cui, J.-Y., Yu, K.-W., Yang, S., Wang, T.-M., et al. (2017). Biosensor-assisted transcriptional regulator engineering for Methylobacterium extorquens AM1 to improve mevalonate synthesis by increasing the acetyl-CoA supply. Metab. Eng. 39, 159-168. doi: 10.1016/j. ymben.2016.11.010

Lim, H. G., Eng, T., Banerjee, D., Alarcon, G., Lau, A. K., Park, M.-R., et al. (2021). Generation of Pseudomonas putida KT2440 strains with efficient utilization of xylose and galactose via adaptive laboratory evolution. ACS Sustain. Chem. Eng. 9, 11512-11523. doi: 10.1021/acssuschemeng. $1 \mathrm{c} 03765$

Lim, H. G., Fong, B., Alarcon, G., Magurudeniya, H. D., Eng, T., Szubin, R., et al. (2020). Generation of ionic liquid tolerant Pseudomonas putida KT2440 strains via adaptive laboratory evolution. Green Chem. 22, 5677-5690. doi: 10.1039/D0GC01663B

Liu, Y., Jiang, X., Cui, Z., Wang, Z., Qi, Q., and Hou, J. (2019). Engineering the oleaginous yeast Yarrowia lipolytica for production of $\alpha$-farnesene. Biotechnol. Biofuels 12:296. doi: 10.1186/s13068-019-1636-Z

Liu, Y., Yan, Z., Lu, X., Xiao, D., and Jiang, H. (2016). Improving the catalytic activity of isopentenyl phosphate kinase through protein coevolution analysis. Sci. Rep. 6:24117. doi: 10.1038/srep24117

Liu, X., Zhu, X., Wang, H., Liu, T., Cheng, J., and Jiang, H. (2020). Discovery and modification of cytochrome P450 for plant natural products biosynthesis. Synth. Syst. Biotechnol. 5, 187-199. doi: 10.1016/j.synbio.2020.06.008

Lund, S., Hall, R., and Williams, G. J. (2019). An artificial pathway for isoprenoid biosynthesis decoupled from native hemiterpene metabolism. ACS Synth. Biol. 8, 232-238. doi: 10.1021/acssynbio.8b00383

Luo, X., Reiter, M. A., d Espaux, L., Wong, J., Denby, C. M., Lechner, A., et al. (2019). Complete biosynthesis of cannabinoids and their unnatural analogues in yeast. Nature 567, 123-126. doi: 10.1038/s41586-019-0978-9

Lyu, Z., Jain, R., Smith, P., Fetchko, T., Yan, Y., and Whitman, W. B. (2016). Engineering the autotroph Methanococcus maripaludis for geraniol production. ACS Synth. Biol. 5, 577-581. doi: 10.1021/acssynbio.5b00267

Martin, V. J. J., Pitera, D. J., Withers, S. T., Newman, J. D., and Keasling, J. D. (2003). Engineering a mevalonate pathway in Escherichia coli for production of terpenoids. Nat. Biotechnol. 21, 796-802. doi: 10.1038/nbt833

Metzger, P., and Largeau, C. (2005). Botryococcus braunii: a rich source for hydrocarbons and related ether lipids. Appl. Microbiol. Biotechnol. 66, 486-496. doi: 10.1007/s00253-004-1779-z

Mi, J., Becher, D., Lubuta, P., Dany, S., Tusch, K., Schewe, H., et al. (2014). De novo production of the monoterpenoid geranic acid by metabolically engineered Pseudomonas putida. Microb. Cell Factories 13:170. doi: 10.1186/ s12934-014-0170-8

Minty, J. J., Singer, M. E., Scholz, S. A., Bae, C.-H., Ahn, J.-H., Foster, C. E., et al. (2013). Design and characterization of synthetic fungal-bacterial consortia for direct production of isobutanol from cellulosic biomass. Proc. Natl. Acad. Sci. U. S. A. 110, 14592-14597. doi: 10.1073/pnas.1218447110

Mohamed, E. T., Werner, A. Z., Salvachúa, D., Singer, C. A., Szostkiewicz, K., Rafael Jiménez-Díaz, M., et al. (2020). Adaptive laboratory evolution of Pseudomonas putida KT2440 improves p-coumaric and ferulic acid catabolism and tolerance. Metab. Eng. Commun. 11:e00143. doi: 10.1016/j.mec.2020. e00143

Morales-Sánchez, D., Martinez-Rodriguez, O. A., and Martinez, A. (2017). Heterotrophic cultivation of microalgae: production of metabolites of commercial interest. J. Chem. Technol. Biotechnol. 92, 925-936. doi: 10.1002/ jctb. 5115

Moser, S., and Pichler, H. (2019). Identifying and engineering the ideal microbial terpenoid production host. Appl. Microbiol. Biotechnol. 103, 5501-5516. doi: 10.1007/s00253-019-09892-y

Moser, S., Strohmeier, G. A., Leitner, E., Plocek, T. J., Vanhessche, K., and Pichler, H. (2018). Whole-cell (+)-ambrein production in the yeast Pichia pastoris. Metab. Eng. Commun. 7:e00077. doi: 10.1016/j.mec.2018.e00077

Nguyen, A. D., Kim, D., and Lee, E. Y. (2020). Unlocking the biosynthesis of sesquiterpenoids from methane via the methylerythritol phosphate pathway in methanotrophic bacteria, using $\alpha$-humulene as a model compound. Metab. Eng. 61, 69-78. doi: 10.1016/j.ymben.2020.04.011

Nikel, P. I., and de Lorenzo, V. (2018). Pseudomonas putida as a functional chassis for industrial biocatalysis: From native biochemistry to transmetabolism. Metab. Eng. 50, 142-155. doi: 10.1016/j.ymben.2018.05.005

Oliver, J. W. K., and Atsumi, S. (2015). A carbon sink pathway increases carbon productivity in cyanobacteria. Metab. Eng. 29, 106-112. doi: 10.1016/j. ymben.2015.03.006

Orita, I., Nishikawa, K., Nakamura, S., and Fukui, T. (2014). Biosynthesis of polyhydroxyalkanoate copolymers from methanol by Methylobacterium extorquens AM1 and the engineered strains under cobalt-deficient conditions. Appl. Microbiol. Biotechnol. 98, 3715-3725. doi: 10.1007/s00253-013-5490-9

Orsi, E., Beekwilder, J., Peek, S., Eggink, G., Kengen, S. W. M., and Weusthuis, R. A. (2020a). Metabolic flux ratio analysis by parallel 13C labeling of isoprenoid biosynthesis in Rhodobacter sphaeroides. Metab. Eng. 57, 228-238. doi: 10.1016/j.ymben.2019.12.004

Orsi, E., Mougiakos, I., Post, W., Beekwilder, J., Dompè, M., Eggink, G., et al. (2020b). Growth-uncoupled isoprenoid synthesis in Rhodobacter sphaeroides. Biotechnol. Biofuels 13:123. doi: 10.1186/s13068-020-01765-1

Orth, J. D., Thiele, I., and Palsson, B. Ø. (2010). What is flux balance analysis? Nat. Biotechnol. 28, 245-248. doi: 10.1038/nbt.1614

Otto, M., Teixeira, P. G., Vizcaino, M. I., David, F., and Siewers, V. (2019). Integration of a multi-step heterologous pathway in Saccharomyces cerevisiae for the production of abscisic acid. Microb. Cell Factories 18:205. doi: 10.1186/ s12934-019-1257-Z

Pang, Y., Zhao, Y., Li, S., Zhao, Y., Li, J., Hu, Z., et al. (2019). Engineering the oleaginous yeast Yarrowia lipolytica to produce limonene from waste cooking oil. Biotechnol. Biofuels 12:241. doi: 10.1186/s13068-019-1580-y

Park, Y.-K., Nicaud, J.-M., and Ledesma-Amaro, R. (2018). The engineering potential of Rhodosporidium toruloides as a workhorse for biotechnological applications. Trends Biotechnol. 36, 304-317. doi: 10.1016/j.tibtech.2017.10.013

Pasternak, Z., Pietrokovski, S., Rotem, O., Gophna, U., Lurie-Weinberger, M. N., and Jurkevitch, E. (2013). By their genes ye shall know them: genomic signatures of predatory bacteria. ISME J. 7, 756-769. doi: 10.1038/ismej.2012.149

Pattanaik, B., Englund, E., Nolte, N., and Lindberg, P. (2020). Introduction of a green algal squalene synthase enhances squalene accumulation in a strain of Synechocystis sp. PCC 6803. Metab. Eng. Commun. 10:e00125. doi: 10.1016/j. mec.2020.e00125

Pham, J. V., Yilma, M. A., Feliz, A., Majid, M. T., Maffetone, N., Walker, J. R., et al. (2019). A review of the microbial production of bioactive natural products and biologics. Front. Microbiol. 10:1404. doi: 10.3389/fmicb.2019.01404

Pramastya, H., Xue, D., Abdallah, I. I., Setroikromo, R., and Quax, W. J. (2021). High level production of amorphadiene using Bacillus subtilis as an optimized terpenoid cell factory. New Biotechnol. 60, 159-167. doi: 10.1016/j. nbt.2020.10.007

Primak, Y. A., Du, M., Miller, M. C., Wells, D. H., Nielsen, A. T., Weyler, W., et al. (2011). Characterization of a feedback-resistant mevalonate kinase from the archaeon Methanosarcina mazei. Appl. Environ. Microbiol. 77, 7772-7778. doi: 10.1128/AEM.05761-11

Rico, J., Duquesne, K., Petit, J.-L., Mariage, A., Darii, E., Peruch, F., et al. (2019). Exploring natural biodiversity to expand access to microbial terpene synthesis. Microb. Cell Factories 18:23. doi: 10.1186/s12934-019-1074-4

Ro, D.-K., Paradise, E. M., Ouellet, M., Fisher, K. J., Newman, K. L., Ndungu, J. M., et al. (2006). Production of the antimalarial drug precursor artemisinic acid in engineered yeast. Nature 440, 940-943. doi: 10.1038/nature04640 
Rodrigues, J. S., and Lindberg, P. (2021). Metabolic engineering of Synechocystis sp. PCC 6803 for improved bisabolene production. Metab. Eng. Commun. 12:e0159. doi: 10.1016/j.mec.2020.e00159

Sánchez-Pascuala, A., Fernández-Cabezón, L., de Lorenzo, V., and Nikel, P. I. (2019). Functional implementation of a linear glycolysis for sugar catabolism in Pseudomonas putida. Metab. Eng. 54, 200-211. doi: 10.1016/j. ymben.2019.04.005

Santos-Merino, M., Torrado, A., Davis, G. A., Röttig, A., Bibby, T. S., Kramer, D. M., et al. (2021). Improved photosynthetic capacity and photosystem I oxidation via heterologous metabolism engineering in cyanobacteria. Proc. Natl. Acad. Sci. U. S. A. 118. doi: 10.1073/pnas.2021523118

Schada von Borzyskowski, L., Sonntag, F., Pöschel, L., Vorholt, J. A., Schrader, J., Erb, T. J., et al. (2018). Replacing the Ethylmalonyl-CoA pathway with the glyoxylate shunt provides metabolic flexibility in the central carbon metabolism of Methylobacterium extorquens AM1. ACS Synth. Biol. 7, 86-97. doi: 10.1021/ acssynbio.7b00229

Schempp, F. M., Drummond, L., Buchhaupt, M., and Schrader, J. (2018). Microbial cell factories for the production of terpenoid flavor and fragrance compounds. J. Agric. Food Chem. 66, 2247-2258. doi: 10.1021/acs.jafc.7b00473

Schrader, J., Schilling, M., Holtmann, D., Sell, D., Filho, M. V., Marx, A., et al. (2009). Methanol-based industrial biotechnology: current status and future perspectives of methylotrophic bacteria. Trends Biotechnol. 27, 107-115. doi: 10.1016/j.tibtech.2008.10.009

Sebesta, J., and Peebles, C. A. (2020). Improving heterologous protein expression in Synechocystis sp. PCC 6803 for alpha-bisabolene production. Metab. Eng. Commun. 10:e00117. doi: 10.1016/j.mec.2019.e00117

Soccol, C. R., Dalmas Neto, C. J., Soccol, V. T., Sydney, E. B., da Costa, E. S. F., Medeiros, A. B. P., et al. (2017). Pilot scale biodiesel production from microbial oil of Rhodosporidium toruloides DEBB 5533 using sugarcane juice: performance in diesel engine and preliminary economic study. Bioresour. Technol. 223, 259-268. doi: 10.1016/j.biortech.2016.10.055

Song, Y., Guan, Z., van Merkerk, R., Pramastya, H., Abdallah, I. I., Setroikromo, R., et al. (2020). Production of squalene in Bacillus subtilis by squalene synthase screening and metabolic engineering. J. Agric. Food Chem. 68, 4447-4455. doi: 10.1021/acs.jafc.0c00375

Song, Y., He, S., Abdallah, I. I., Jopkiewicz, A., Setroikromo, R., van Merkerk, R., et al. (2021). Engineering of multiple modules to improve amorphadiene production in Bacillus subtilis using CRISPR-Cas9. J. Agric. Food Chem. 69, 4785-4794. doi: 10.1021/acs.jafc.1c00498

Sonntag, F., Kroner, C., Lubuta, P., Peyraud, R., Horst, A., Buchhaupt, M., et al. (2015). Engineering Methylobacterium extorquens for de novo synthesis of the sesquiterpenoid $\alpha$-humulene from methanol. Metab. Eng. 32, 82-94. doi: 10.1016/j.ymben.2015.09.004

Sun, W., Xue, H., Liu, H., Lv, B., Yu, Y., Wang, Y., et al. (2020). Controlling chemo- and regioselectivity of a plant $\mathrm{P} 450$ in yeast cell toward rare licorice triterpenoid biosynthesis. ACS Catal. 10, 4253-4260. doi: 10.1021/ acscatal.0c00128

Sun, T., Yu, Y., Wang, K., Ledesma-Amaro, R., and Ji, X.-J. (2021). Engineering Yarrowia lipolytica to produce fuels and chemicals from xylose: a review. Bioresour. Technol. 337:125484. doi: 10.1016/j.biortech.2021.125484

Sun, J., Zhang, C., Nan, W., Li, D., Ke, D., and Lu, W. (2018). Glycerol improves heterologous biosynthesis of betulinic acid in engineered Yarrowia lipolytica. Chem. Eng. Sci. 196, 82-90. doi: 10.1016/j.ces.2018.10.052

Thomas, S. T., Louie, G. V., Lubin, J. W., Lundblad, V., and Noel, J. P. (2019). Substrate specificity and engineering of mevalonate 5-phosphate decarboxylase. ACS Chem. Biol. 14, 1767-1779. doi: 10.1021/acschembio.9b00322

Tian, T., Kang, J. W., Kang, A., and Lee, T. S. (2019). Redirecting metabolic flux via combinatorial multiplex CRISPRi-mediated repression for isopentenol production in Escherichia coli. ACS Synth. Biol. 8, 391-402. doi: 10.1021/ acssynbio. 8 b00429

Tsuruta, H., Paddon, C. J., Eng, D., Lenihan, J. R., Horning, T., Anthony, L. C., et al. (2009). High-level production of amorpha-4,11-diene, a precursor of the antimalarial agent artemisinin, in Escherichia coli. PLoS One 4:e4489. doi: 10.1371/journal.pone.0004489

Vannice, J. C., Skaff, D. A., Keightley, A., Addo, J. K., Wyckoff, G. J., and Miziorko, H. M. (2014). Identification in Haloferax volcanii of phosphomevalonate decarboxylase and isopentenyl phosphate kinase as catalysts of the terminal enzyme reactions in an archaeal alternate mevalonate pathway. J. Bacteriol. 196, 1055-1063. doi: 10.1128/JB.01230-13
Vickers, C. E., Williams, T. C., Peng, B., and Cherry, J. (2017). Recent advances in synthetic biology for engineering isoprenoid production in yeast. Curr. Opin. Chem. Biol. 40, 47-56. doi: 10.1016/j.cbpa.2017.05.017

Volke, D. C., Rohwer, J., Fischer, R., and Jennewein, S. (2019). Investigation of the methylerythritol 4-phosphate pathway for microbial terpenoid production through metabolic control analysis. Microb. Cell Factories 18:192. doi: 10.1186/ s12934-019-1235-5

von Reuss, S., Domik, D., Lemfack, M. C., Magnus, N., Kai, M., Weise, T., et al. (2018). Sodorifen biosynthesis in the rhizobacterium serratia plymuthica involves methylation and cyclization of MEP-derived farnesyl pyrophosphate by a SAM-dependent C-methyltransferase. J. Am. Chem. Soc. 140, 11855-11862. doi: $10.1021 /$ jacs. 8 b08510

Vranová, E., Coman, D., and Gruissem, W. (2012). Structure and dynamics of the isoprenoid pathway network. Mol. Plant 5, 318-333. doi: 10.1093/mp/ sss015

Wang, X., Liu, W., Xin, C., Zheng, Y., Cheng, Y., Sun, S., et al. (2016). Enhanced limonene production in cyanobacteria reveals photosynthesis limitations. Proc. Natl. Acad. Sci. U. S. A. 113, 14225-14230. doi: 10.1073/ pnas. 1613340113

Wang, P., Wei, W., Ye, W., Li, X., Zhao, W., Yang, C., et al. (2019). Synthesizing ginsenoside $\mathrm{Rh} 2$ in Saccharomyces cerevisiae cell factory at high-efficiency. Cell Discov. 5:5. doi: 10.1038/s41421-018-0075-5

Ward, V. C. A., Chatzivasileiou, A. O., and Stephanopoulos, G. (2018). Metabolic engineering of Escherichia coli for the production of isoprenoids. FEMS Microbiol. Lett. 365. doi: 10.1093/femsle/fny079

Wichmann, J., Baier, T., Wentnagel, E., Lauersen, K. J., and Kruse, O. (2018). Tailored carbon partitioning for phototrophic production of (E)- $\alpha$-bisabolene from the green microalga Chlamydomonas reinhardtii. Metab. Eng. 45, 211-222. doi: 10.1016/j.ymben.2017.12.010

Wong, J., de Rond, T., d Espaux, L., van der Horst, C., Dev, I., Rios-Solis, L., et al. (2018). High-titer production of lathyrane diterpenoids from sugar by engineered Saccharomyces cerevisiae. Metab. Eng. 45, 142-148. doi: 10.1016/j. ymben.2017.12.007

Wriessnegger, T., Augustin, P., Engleder, M., Leitner, E., Müller, M., Kaluzna, I., et al. (2014). Production of the sesquiterpenoid (+)-nootkatone by metabolic engineering of Pichia pastoris. Metab. Eng. 24, 18-29. doi: 10.1016/j. ymben.2014.04.001

Wu, W., and Maravelias, C. T. (2018). Synthesis and techno-economic assessment of microbial-based processes for terpenes production. Biotechnol. Biofuels 11:294. doi: 10.1186/s13068-018-1285-7

Xiao, H., Zhang, Y., and Wang, M. (2019). Discovery and engineering of cytochrome p450s for terpenoid biosynthesis. Trends Biotechnol. 37, 618-631. doi: $10.1016 /$ j.tibtech.2018.11.008

Xie, D., Jackson, E. N., and Zhu, Q. (2015). Sustainable source of omega-3 eicosapentaenoic acid from metabolically engineered Yarrowia lipolytica: from fundamental research to commercial production. Appl. Microbiol. Biotechnol. 99, 1599-1610. doi: 10.1007/s00253-014-6318-y

Yadav, V. G., De Mey, M., Lim, C. G., Ajikumar, P. K., and Stephanopoulos, G. (2012). The future of metabolic engineering and synthetic biology: towards a systematic practice. Metab. Eng. 14, 233-241. doi: 10.1016/j.ymben.2012.02.001

Yaegashi, J., Kirby, J., Ito, M., Sun, J., Dutta, T., Mirsiaghi, M., et al. (2017). Rhodosporidium toruloides: a new platform organism for conversion of lignocellulose into terpene biofuels and bioproducts. Biotechnol. Biofuels 10:241. doi: 10.1186/s13068-017-0927-5

Yang, C., Gao, X., Jiang, Y., Sun, B., Gao, F., and Yang, S. (2016). Synergy between methylerythritol phosphate pathway and mevalonate pathway for isoprene production in Escherichia coli. Metab. Eng. 37, 79-91. doi: 10.1016/j. ymben.2016.05.003

Yang, J., Im, Y., Kim, T. H., Lee, M. J., Cho, S., Na, J.-G., et al. (2020). Engineering Pseudomonas putida KT2440 to convert 2,3-butanediol to mevalonate. Enzym. Microb. Technol. 132:109437. doi: 10.1016/j. enzmictec.2019.109437

Yang, X., Jiang, Y., Yang, J., He, J., Sun, J., Chen, F., et al. (2015). Prenylated flavonoids, promising nutraceuticals with impressive biological activities. Trends Food Sci. Technol. 44, 93-104. doi: 10.1016/j.tifs.2015.03.007

Yang, J., Son, J. H., Kim, H., Cho, S., Na, J.-G., Yeon, Y. J., et al. (2019). Mevalonate production from ethanol by direct conversion through acetylCoA using recombinant Pseudomonas putida, a novel biocatalyst for terpenoid production. Microb. Cell Factories 18:168. doi: 10.1186/s12934-019-1213-y 
Yao, F., Liu, S.-C., Wang, D.-N., Liu, Z.-J., Hua, Q., and Wei, L.-J. (2020). Engineering oleaginous yeast Yarrowia lipolytica for enhanced limonene production from xylose and lignocellulosic hydrolysate. FEMS Yeast Res. 20:foaa046. doi: 10.1093/femsyr/foaa046

Ye, R.-P., Ding, J., Gong, W., Argyle, M. D., Zhong, Q., Wang, Y., et al. (2019). $\mathrm{CO} 2$ hydrogenation to high-value products via heterogeneous catalysis. Nat. Commun. 10:5698. doi: 10.1038/s41467-019-13638-9

Ye, R. W., Yao, H., Stead, K., Wang, T., Tao, L., Cheng, Q., et al. (2007). Construction of the astaxanthin biosynthetic pathway in a methanotrophic bacterium Methylomonas sp. strain 16a. J. Ind. Microbiol. Biotechnol. 34, 289-299. doi: 10.1007/s10295-006-0197-x

Yishai, O., Lindner, S. N., Gonzalez de la Cruz, J., Tenenboim, H., and Bar-Even, A. (2016). The formate bio-economy. Curr. Opin. Chem. Biol. 35, 1-9. doi: 10.1016/j.cbpa.2016.07.005

Yook, S. D., Kim, J., Gong, G., Ko, J. K., Um, Y., Han, S. O., et al. (2020). High-yield lipid production from lignocellulosic biomass using engineered xylose-utilizing Yarrowia lipolytica. Glob. Change Biol. Bioenergy. 12, 670-679. doi: $10.1111 /$ gcbb.12699

Yoshida, R., Yoshimura, T., and Hemmi, H. (2020). Reconstruction of the "Archaeal" Mevalonate pathway from the Methanogenic Archaeon Methanosarcina mazei in Escherichia coli cells. Appl. Environ. Microbiol. 86, e02889-e02819. doi: 10.1128/AEM.02889-19

Yu, F., Okamoto, S., Harada, H., Yamasaki, K., Misawa, N., and Utsumi, R. (2011). Zingiber zerumbet CYP71BA1 catalyzes the conversion of $\alpha$-humulene to 8-hydroxy- $\alpha$-humulene in zerumbone biosynthesis. Cell. Mol. Life Sci. 68, 1033-1040. doi: 10.1007/s00018-010-0506-4

Yuan, L. Z., Rouvière, P. E., Larossa, R. A., and Suh, W. (2006). Chromosomal promoter replacement of the isoprenoid pathway for enhancing carotenoid production in E. coli. Metab. Eng. 8, 79-90. doi: 10.1016/j.ymben. 2005.08.005

Zhang, X., Guan, H., Dai, Z., Guo, J., Shen, Y., Cui, G., et al. (2015). Functional analysis of the isopentenyl diphosphate isomerase of salvia miltiorrhiza via color complementation and RNA interference. Molecules 20, 20206-20218. doi: $10.3390 /$ molecules 201119689

Zhang, C., Liu, J., Zhao, F., Lu, C., Zhao, G.-R., and Lu, W. (2018). Production of sesquiterpenoid zerumbone from metabolic engineered Saccharomyces cerevisiae. Metab. Eng. 49, 28-35. doi: 10.1016/j.ymben.2018.07.010
Zhang, X., Wang, D., Duan, Y., Zheng, X., Lin, Y., and Liang, S. (2020). Production of lycopene by metabolically engineered Pichia pastoris. Biosci. Biotechnol. Biochem. 84, 463-470. doi: 10.1080/09168451.2019.1693250

Zheng, Y., Liu, Q., Li, L., Qin, W., Yang, J., Zhang, H., et al. (2013). Metabolic engineering of Escherichia coli for high-specificity production of isoprenol and prenol as next generation of biofuels. Biotechnol. Biofuels 6:57. doi: 10.1186/1754-6834-6-57

Zhou, F., and Pichersky, E. (2020). More is better: the diversity of terpene metabolism in plants. Curr. Opin. Plant Biol. 55, 1-10. doi: 10.1016/j. pbi.2020.01.005

Zhou, K., Qiao, K., Edgar, S., and Stephanopoulos, G. (2015). Distributing a metabolic pathway among a microbial consortium enhances production of natural products. Nat. Biotechnol. 33, 377-383. doi: 10.1038/nbt.3095

Zhou, K., Zou, R., Zhang, C., Stephanopoulos, G., and Too, H.-P. (2013). Optimization of amorphadiene synthesis in bacillus subtilis via transcriptional, translational, and media modulation. Biotechnol. Bioeng. 110, 2556-2561. doi: 10.1002/bit.24900

Zu, Y., Prather, K. L., and Stephanopoulos, G. (2020). Metabolic engineering strategies to overcome precursor limitations in isoprenoid biosynthesis. Curr. Opin. Biotechnol. 66, 171-178. doi: 10.1016/j.copbio.2020.07.005

Conflict of Interest: The authors declare that they have no known competing financial interests or personal relationships that could have appeared to influence the work reported in this paper.

Publisher's Note: All claims expressed in this article are solely those of the authors and do not necessarily represent those of their affiliated organizations, or those of the publisher, the editors and the reviewers. Any product that may be evaluated in this article, or claim that may be made by its manufacturer, is not guaranteed or endorsed by the publisher.

Copyright (c) 2021 Carruthers and Lee. This is an open-access article distributed under the terms of the Creative Commons Attribution License (CC BY). The use, distribution or reproduction in other forums is permitted, provided the original author(s) and the copyright owner(s) are credited and that the original publication in this journal is cited, in accordance with accepted academic practice. No use, distribution or reproduction is permitted which does not comply with these terms. 